\title{
Stochastic Hybrid Systems with Renewal Transitions
}

\author{
Duarte Antunes, João P. Hespanha, and Carlos Silvestre ${ }^{* \dagger}$
}

August 27, 2009

\begin{abstract}
We consider Stochastic Hybrid Systems (SHSs) for which the lengths of times that the system stays in each mode are independent random variables with given distributions. We propose an analysis framework based on a set of Volterra renewal-type equations, which allows us to compute any statistical moment of the state of the SHS. Moreover, we provide necessary and sufficient conditions for various stability notions, and determine the exponential decay or increase rate at which the expected value of the energy of the system converges to zero or to infinity, respectively. The applicability of the results is illustrated in a networked control problem considering independently distributed intervals between data transmissions and delays.
\end{abstract}

\section{Introduction}

A Hybrid System is a system with both continuous dynamics and discrete logic. Its execution is specified by the dynamic equations of the continuous state, a set of rules governing the transitions between discrete modes, and reset maps determining jumps of the state at transition times.

Stochastic Hybrid Systems (SHSs) introduce randomness in the execution of the Hybrid System. As surveyed in [1], various models of SHSs have been proposed differing on where randomness comes into play. In [2] and [3], the continuous dynamics are driven by a Wiener process. In [3] and [4], transitions are triggered by stochastic events, and [4] allows transitions for which the next state is chosen according to a given distribution. See also [5], [6], [7] and the references therein for additional work on stochastic hybrid systems.

The present work follows closely the definition of a SHS given in [5], where randomness arises solely from the fact that the rate at which stochastically spaced transitions occur is allowed to depend on both the continuous state and discrete modes. However, as noted in [5] this model can capture systems where the next state is chosen according to a given distribution, the dynamics is driven by a Wiener process, and discrete transitions are triggered by deterministic

\footnotetext{
* \{dantunes,cjs $\} @$ isr.ist.utl.pt, hespanha@ece.ucsb.edu, Institute for Systems and Robotics, University of California, Santa Barbara

†This research is partially supported by the National Science Foundation under the grants CNS-0720842, ECCS-0725485 and ECCS-0835847
} 
conditions on the state, e.g, guards being crossed. In [5], a formula is provided for the extended generator of the Markov process associated with the SHS, which, as explained in [4, Ch.1], completely characterizes the Markov process. The generator can be used to compute expectations and probabilities by using the Dynkin's formula, in some problems with special structure. This approach is followed in [4] for various cases of interest. It is also the starting point of the method proposed in [5] to compute the moments of the sending rate for an on-off TCP flow model.

The approach based on the Dynkin's formula is also used in [8, Ch. 2] to compute the moments of the state of a Markov Jump Linear System (MJLS). This class of systems can be viewed as a special case of a stochastic hybrid system, in which the length of times that the system stays in each discrete mode are independent exponentially distributed random variables, with a mean that may depend on the discrete mode.

In the present work we consider stochastic hybrid system with linear dynamics, linear reset maps, and for which the lengths of times that the system stays in each mode are independent arbitrarily distributed random variables, whose distributions may depend on the discrete mode. The process that combines the transition times and the discrete mode is called a Markov Renewal process [9], which motivated us to refer to these systems as stochastic hybrid systems with renewal transitions. This class of systems can be viewed as a special case of the SHS model in [5], or as a generalization of a MJLS.

A key challenge in the analysis of the systems considered here, lies in the fact that the discrete component of the state is only a semi-Markov process [9], instead of a Markov chain, which would be in the case of MJLS. This prevents the direct use of approaches based on the Dynkyn's formula to compute the statistical moments of the continuous state of the system, which is possible for exponential distributions in the durations of each mode [8, Ch. 2]. For nonexponential distributions, an approximation is proposed in [8, Ch. 8] that relies on the fact that a more general distribution can be approximated by the sum of exponential distributions. However, aside from introducing approximation errors, this approach requires a very large number of discrete modes when the distributions needs to be approximated as the sum of several exponentials.

Inspired by our recent work on impulsive renewal systems [10], the approach followed here to analyze SHS with renewal transitions is based on a set of Volterra renewal-type equations. This allows us to give expressions for any moment of the state of the SHS. Moreover, we characterize the asymptotic behavior of the system by providing necessary and sufficient conditions for various stability notions in terms of LMI, algebraic and Nyquist criterium conditions. We also determine the decay or increase rate at which the expected value of the energy of the system converges exponentially to zero or to infinity, depending on whether or not the system is mean exponentially stable.

The applicability of our theoretical results is illustrated in a networked control problem. We consider independently distributed intervals between data transmissions of the control signal in a feedback-loop, which, as observed in [10], is a reasonable assumption in networked control systems utilizing CSMA protocols. The impulsive renewal systems considered in [10] did not permit us to consider the effect of network induced delays, which is now possible with SHSs with renewal transitions.

The remainder of the paper is organized as follows. SHSs with renewal 
transitions are defined in Section 2 and the connection with previous models of SHSs is addressed. In Section 3, we compute the statistical state moments of the SHS, and in Section 4 we provide results related to the asymptotic behavior of the SHS. Section 6 addresses the application of the results to networked control systems. In Section 7 we draw final conclusions and address future work.

Notation and Preliminaries: For dimensionally compatible matrices $A$ and $B$, we define $(A, B):=\left[A^{\prime} B^{\prime}\right]^{\prime}$. The $n \times n$ identity and zero matrices are denoted by $I_{n}$ and $0_{n}$, respectively. For a given matrix $A$, its transpose is denoted by $A^{\prime}$, its spectral radius by $\sigma(A)$, and an eigenvalue by $\lambda_{i}(A)$. The Kronecker product is denoted by $\otimes$. For a complex number $z, \Re[z]$ and $\Im[z]$ denote the real and complex parts of $z$, respectively. The notation $x\left(t_{k}^{-}\right)$indicates the limit from the left of a function $x(t)$ at the point $t_{k}$. The probability space is denoted by $(\boldsymbol{\Omega}, \mathbf{B}, \mathbf{P})$ and the expected value is denoted by $\mathbb{E}($.$) . The indicator function of a$ set $A$ is denoted by $\mathbf{1}_{x \in A}$, which equals 1 if $x \in A$ and 0 otherwise. A probability distribution $F(\tau)$ with support on a given interval $[0, T], T \in \mathbb{R}_{>0} \cup\{+\infty\}$ takes the form $F=F^{c}+F^{d}$, where $F^{c}$ is an absolutely continuous function $F^{c}(\tau)=$ $\int_{0}^{\tau} f(r) d r$, for some density function $f(r) \geq 0, \forall_{r \geq 0}$, and $F^{d}$ is a piecewise constant increasing function that captures possible atom points $\left\{b^{j}>0\right\}$ where the cumulative distribution places mass $\left\{w^{j}\right\}$. Notice that, the integral with respect to the monotone function $F$, in general parametric on some variable $y$, is then given by

$$
G(y)=\int_{0}^{T} W(y, \tau) F(d \tau)=G^{c}(y)+G^{d}(y),
$$

where $G^{c}(y)=\int_{0}^{T} W(y, \tau) f(\tau) d \tau, G^{d}(y)=\sum_{j} w^{j} W\left(y, b^{j}\right)$, and $W(y, \tau)$ is generally a matrix-valued function that depends on the integration variable $\tau$ and generally also on some parameter $y$. We write $G(y)<\infty$ to say that $G(y)$ exists for a given $y$, i.e., the integral exists as an absolutely convergent Lebesgue integral.

\section{SHS with Renewal Transitions}

A SHS with renewal transitions, is defined by a linear differential equation

$$
\dot{x}(t)=A_{q(t)} x(t), \quad x(0)=x_{0}, q(0)=q_{0}, t_{0}=0,
$$

where $x(t) \in \mathbb{R}^{n}$, and $q(t) \in \mathcal{Q}:=\left\{1, \ldots, n_{q}\right\}$; a family of $n_{l}$ discrete transition/reset maps

$$
\begin{aligned}
\left(q\left(t_{k}\right), x\left(t_{k}\right)\right)=\left(\xi_{l}\left(q\left(t_{k}^{-}\right)\right), J_{q\left(t_{k}^{-}\right), l} x\left(t_{k}^{-}\right)\right), & \\
l & \quad \in \mathcal{L}:=\left\{1, \ldots, n_{l}\right\},
\end{aligned}
$$

where $\xi_{l}$ is a map $\xi_{l}: \mathcal{Q} \mapsto \mathcal{Q}$ and $J_{q\left(t_{k}^{-}\right), l}$ belongs to the set $\left\{J_{i, l} \in \mathbb{R}^{n \times n}, i \in\right.$ $\mathcal{Q}, l \in \mathcal{L}\}$; and a family of transition distributions

$$
F_{i, l}(\tau), \quad i \in \mathcal{Q}, \quad l \in \mathcal{L}
$$

Between transition times $t_{k}$, the discrete mode $q$ remains constant whereas the continuous state $x$ flows according to (2). At transition times, the continuous 
state and discrete mode of the SHS are reset according to (3). The intervals between transition times are independent random variables determined by the transition distributions (4) as follows. A transition distribution can be either a probability distribution or identically zero. In the former case, $F_{i, l}$ is the probability distribution of the random time that transition $l \in \mathcal{L}$ takes to trigger in the state $q(t)=i \in \mathcal{Q}$. The next transition time is determined by the minimum triggering times of the transitions associated with state $q(t)=i \in \mathcal{Q}$. When $F_{i, l}(\tau)=0, \forall_{\tau}$, the transition $l$ does not trigger in the state $i \in \mathcal{Q}$, i.e., the reset maps may be specified only for a subset of the states.

When the transition distributions have discrete parts, different transitions may trigger at the same time with probability different than zero, leading to an ambiguity in choosing the next state. Thus, to guarantee that with probability 1 , this does not occur, we assume the following. Suppose that the transitions distributions $F_{i, l}$ have support on a given interval $\left[0, T_{i, l}\right], T_{i, l} \in \mathbb{R}_{>0} \cup\{+\infty\}$, and can be decomposed as $F_{i, l}=F_{i, l}^{c}+F_{i, l}^{d}$, where $F_{i, l}^{c}(\tau)=\int_{0}^{\tau} f_{i, l}(r) d r, f_{i, l}(r) \geq$ $0, \forall_{r}$, and $F_{i, l}^{d}$ captures atom points $\left\{0<b_{i, l}^{j} \leq T_{i, l}\right\}$ with mass $\left\{w_{i, l}^{j}\right\}$.

Assumption 1 For every $i \in \mathcal{Q}$, the (possible) atom points of the transition distributions verify $b_{i, l_{1}}^{j} \neq b_{i, l_{2}}^{\kappa}$ for $l_{1} \neq l_{2}, l_{1} \in \mathcal{L}, l_{2} \in \mathcal{L}, \forall_{j, \kappa}$.

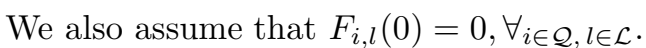

The construction of a sample path of the SHS with renewal transitions can be described as follows.

1. Set $k=0, t_{0}=0,\left(q\left(t_{k}\right), x\left(t_{k}\right)\right)=\left(q_{0}, x_{0}\right)$.

2. For every $j \in \mathcal{L}$, obtain $\bar{h}_{k}^{j}$ as a realization of a random variable distributed according to $F_{q\left(t_{k}\right), j}$, if $F_{q\left(t_{k}\right), j}$ is not identically zero, and set $\bar{h}_{k}^{j}=\infty$ otherwise.

3. Take $h_{k}=\min \left\{\bar{h}_{k}^{j}, j \in \mathcal{L}\right\}$ and set the next transition time to $t_{k+1}=$ $t_{k}+h_{k}$. The state of the SHS in the interval $t \in\left[t_{k}, t_{k+1}\right)$ is given by $(q(t), x(t))=\left(q\left(t_{k}\right), e^{A_{q\left(t_{k}\right)} t} x\left(t_{k}\right)\right)$.

4. In the case where $t_{k+1}<\infty$, take $l_{k}=j: h_{k}=\bar{h}_{k}^{j}$ and update the state according to

$$
\left(q\left(t_{k+1}\right), x\left(t_{k+1}\right)\right)=\left(\xi_{l_{k}}\left(q\left(t_{k+1}^{-}\right)\right), J_{q\left(t_{k+1}^{-}\right), l_{k}} x\left(t_{k+1}^{-}\right)\right) .
$$

Set $k=k+1$ and repeat the construction from the step 2 .

Notice that due to the Assumption 1, in step three the probability of existing two or more indexes $j$ for which $h_{k}=\bar{h}_{k}^{j}$ is zero.

\section{Connection with previous SHSs}

In general the state $(x, q)$ in $(2),(3)$ is not a Markov process, but it can be made Markov by introducing an auxiliar variable $\tau(t):=t-t_{k}$, which keeps track of the time elapsed since the last transition, and augmenting the continuous state to $\mathbf{x}:=(x, \tau)$. The resulting state can be viewed as the state of the class of SHS considered in [5]. In [5], a SHS is defined by a differential equation

$$
\dot{\mathbf{x}}(t)=f(q(t), \mathbf{x}(t), t), \quad x(0)=x_{0}, q(0)=q_{0}, t_{0}=0,
$$


a family of $n_{l}$ discrete transition/reset maps

$$
\left(q\left(t_{k}\right), \mathbf{x}\left(t_{k}\right)\right)=\phi_{l}\left(q\left(t_{k}^{-}\right), \mathbf{x}\left(t_{k}^{-}\right), t\right), \quad l \in\left\{1, \ldots, n_{l}\right\},
$$

and a family of $n_{l}$ transition intensities

$$
\lambda_{l}(q(t), \mathbf{x}(t), t), \quad l \in\left\{1, \ldots, n_{l}\right\} .
$$

The transition intensities determine the rate at which transitions occur. If we consider that they depend on $\tau$ and $q$ but not on $x$ and $t$, the transition intensities come down to hazard rates, i.e., they have a one-to-one correspondence with absolutely continuous transition distributions given by

$$
\lambda_{l}(i, \tau)=\frac{\dot{F}_{i, l}(\tau)}{1-F_{i, l}(\tau)}, \tau \in\left[0, T_{i, l}\right), F_{i, l}(0)=0 .
$$

Thus, provided that the transition distributions are absolutely continuous, the SHS with renewal transitions is a special case of the class of SHS considered in [5], since we can consider $\mathbf{x}=(x, \tau)$, where the dynamics of $\mathbf{x}$ take the form (2) and $\dot{\tau}=1$, and the reset map take the form (3) and $\tau\left(t_{k}\right)=0$. Note that in the present paper, we do not need to restrict the transition distributions to be absolutely continuous.

Two of the three generalizations discussed in [5] still apply to SHS with renewal transitions. Namely, we could generalize SHS with renewal transitions to the case where the dynamics are driven by a Wiener process and the next state is chosen according to a given distribution, where the last generalization further requiring the transition distributions to be absolutely continuous. However, we cannot model the case where discrete transitions are triggered by deterministic conditions on the state, e.g, guards being crossed. Moreover, note that, exogenous inputs described by linear systems can be easily incorporated in both dynamic equations and reset maps, e.g., $\dot{x}_{s}(t)=A_{i} x_{s}(t)+B_{i} r(t)$, where the exogenous input is described by $\dot{x}_{r}(t)=A_{r} x_{r}(t), r(t)=C_{r} x_{r}(t)$, can be written in the form (2) considering $x=\left(x_{s} x_{r}\right)$.

Another related class of systems are MJLSs (see [8]), where the continuous dynamics depend on a discrete mode which evolves according to a continuoustime Markov Chain with $n_{M C}$ states, i.e., the intervals between mode transitions are exponentially distributed. Note that this is a special case of a SHS with renewal transitions and can be modeled, for instance, by $n_{q}=n_{M C}, n_{l}=n_{q}$,

$$
F_{i, l}(\tau)=\left\{\begin{array}{l}
\pi_{i l} e^{-\pi_{i l} \tau}, \tau \geq 0, \text { if } i \neq l, \\
0 \text { if } i=l
\end{array}\right.
$$

and $J_{i, l}=I_{n}, \forall_{i \in \mathcal{Q}, l \in \mathcal{L}}$.

\section{State Moments}

Consider an $m$-th order uncentered moment of the state of the SHS with renewal transitions, i.e.,

$$
\mathbb{E}\left[x_{i_{1}}(t)^{m_{1}} x_{i_{2}}(t)^{m_{2}} \ldots x_{i_{r}}(t)^{m_{r}}\right]
$$


where $1 \leq i_{1}, \ldots, i_{r} \leq n, \sum_{j=1}^{r} m_{j}=m, m_{j}>0$ and $x_{i}(t) \in \mathbb{R}$ denotes the $i$ th component of $x(t)$. Let $A^{(m)}, m \geq 0$, denote the pth fold kronecker product of a matrix $A$ with itself $\underbrace{A \otimes A \cdots \otimes A}_{m}, A^{0}:=I$ and recall that (cf. [11])

$$
(A \otimes B)(C \otimes D)=(A C) \otimes(B D) .
$$

Using (6), we can write (5) as

$$
\mathbb{E}\left[c^{\prime} x(t)^{(m)}\right]
$$

where

$$
c=e_{i_{1}}^{\left(m_{1}\right)} \otimes e_{i_{2}}^{\left(m_{2}\right)} \otimes \ldots e_{i_{r}}^{\left(m_{r}\right)},
$$

and $e_{j} \in \mathbb{R}^{n}$ is the canonical basis vector whose components equal zero except component $j$ that equals 1 . Furthermore, let $\Phi_{j}(t)$ denote the transition matrix of the SHS starting at the discrete mode $q_{0}=j$, i.e., $x(t)=\Phi_{q_{0}}(t) x_{0}$ where

$$
\Phi_{j}(t)=e^{A_{q(t)}\left(t-t_{r}\right)} \ldots J_{\xi_{l_{0}}(j), \xi_{l_{1}}\left(\xi_{l_{0}}(j)\right)} e^{A_{\xi_{l_{0}}(j)}} J_{j, \xi_{l_{0}}(j)} e^{A_{j} t},
$$

$r=\max \left\{k \in \mathbb{Z}_{\geq 0}: t_{k} \leq t\right\}$, and let

$$
z_{i}^{m}(t):=\mathbb{E}\left[\left(\Phi_{i}(t)^{\prime}\right)^{(m)} c\right], \quad i \in \mathcal{Q} .
$$

We can express (5), or equivalently (7), in terms of $z_{i}^{m}(t), i \in \mathcal{Q}$. In fact, for an initial condition $\left(x_{0}, q_{0}\right)$,

$$
\begin{aligned}
\mathbb{E}\left[c^{\prime} x(t)^{(m)}\right] & =\mathbb{E}\left[\left(x(t)^{\prime}\right)^{(m)} c\right] \\
& =\left(x_{0}^{\prime}\right)^{(m)} \mathbb{E}\left[\left(\Phi_{q_{0}}(t)^{\prime}\right)^{(m)} c\right]=\left(x_{0}^{\prime}\right)^{(m)} z_{q_{0}}^{m}(t),
\end{aligned}
$$

where we used (6).

The next theorem states that

$$
z^{m}(t):=\left(z_{1}^{m}(t), \ldots, z_{n_{q}}^{m}(t)\right)
$$

verifies a Volterra renewal-type equation. We define

$$
K_{m}\left(z^{m}(t)\right):=\left(K_{m, 1}\left(z^{m}(t)\right), \ldots, K_{m, n_{q}}\left(z^{m}(t)\right)\right),
$$

where $K_{m, i}$ is a convolution operator defined as

$$
\begin{aligned}
& K_{m, i}\left(z^{m}(t)\right):= \\
& \quad \sum_{l=1}^{n_{l}} \int_{0}^{t}\left(E_{i, l}(\tau)^{\prime}\right)^{(m)} z_{\xi_{l}(i)}^{m}(t-\tau) \frac{S_{i}(\tau)}{S_{i, l}(\tau)} F_{i, l}(d \tau),
\end{aligned}
$$

where $i \in \mathcal{Q}, S_{i}(\tau):=\prod_{l=1}^{n_{l}} S_{i, l}(\tau), S_{i, l}(\tau):=1-F_{i, l}(\tau)$ and $E_{i, l}(\tau):=J_{i, l} e^{A_{i} \tau}$.

Theorem 2 The function (11) satisfies

$$
z^{m}(t)=K_{m}\left(z^{m}(t)\right)+h^{m}(t), \quad t \geq 0
$$

where $h^{m}(t):=\left(h_{1}^{m}(t), \ldots, h_{n_{q}}^{m}(t)\right)$, and

$$
h_{i}^{m}(t)=\left(e^{A_{i}^{\prime} t}\right)^{(m)} c S_{i}(t), i \in \mathcal{Q} .
$$


As stated in the next result, we can obtain an explicit expression for $z^{m}(t)$, and therefore to any $m$ th order uncentered moment of the state of the SHS due to (10). We denote by $K_{m}^{j}$ the composite operator obtained by applying $j$ times $K_{m}$, e.g., $K_{m}^{2}(y(t))=K_{m}\left(K_{m}(y(t))\right)$ and say that a function defined in $\mathbb{R}_{\geq 0}$ is locally integrable if its integral is finite in every compact subset of $\mathbb{R}_{\geq 0}$.

Theorem 3 There exists a unique locally integrable solution to (13) given by

$$
z^{m}(t)=\sum_{j=1}^{\infty} K_{m}^{j}\left(h^{m}(t)\right)+h^{m}(t), \quad t \geq 0 .
$$

Although (15) provides an explicit expression for $z^{m}(t)$, in general it is difficult to solve the series that appears in this expression. If one is interested in plotting the solution to the Volterra equation, or the moments of the state, it is typically easier to use a numerical method. Efficient numerical methods can be found in [12]. However, in some cases one can obtain a manageable expression by using Laplace transforms [13].

\section{Proofs}

Proof. (of Theorem 2) Consider an initial condition $q_{0}=i, i \in \mathcal{Q}$ and a given time $t$ and partition the probability space $\boldsymbol{\Omega}$ into $\boldsymbol{\Omega}=\left[t_{1} \leq t\right] \cup\left[t_{1}>t\right]$. We can further partition $\left[t_{1} \leq t\right]$ into $\left[t_{1} \leq t\right]=\cup_{l=1}^{n_{l}} B_{l}(t) \cup B_{0}(t)$, where $B_{0}(t)$ is the event of two transitions triggering at the same time in the interval $[0, t]$, which has probability zero due to Assumption 1 , and $B_{l}(t)$ is the event of the transition $l$ being the first to trigger in the interval $[0, t]$, i.e., $B_{l}(t)=\left[\min \left\{\bar{h}_{0}^{j}, j \in \mathcal{L}\right\}=\right.$ $\left.\bar{h}_{0}^{l}=t_{1} \leq t\right] \wedge\left[\bar{h}_{0}^{j}>\bar{h}_{0}^{l}, l \neq j\right]$. Notice that, since the initial state is $q_{0}=i, \bar{h}_{0}^{j}$ is distributed according to $F_{i, j}$, for a given $j \in \mathcal{L}$ for which $F_{i, j}$ is not identically zero. When transition $l$ does not trigger in state $q_{0}=i$, the event $B_{l}(t)$ is empty in which case $F_{i, j}$ is identically zero. Using this partition we can write

$$
\begin{aligned}
\mathbb{E}\left[\left(\Phi_{i}(t)^{\prime}\right)^{(m)} c\right] & =\mathbb{E}\left[\left(\Phi_{i}(t)^{\prime}\right)^{(m)} c \mathbf{1}_{\left[t_{1}>t\right]}\right] \\
& +\sum_{l=1}^{n_{l}} \mathbb{E}\left[\left(\Phi_{i}(t)^{\prime}\right)^{(m)} c \mathbf{1}_{B_{l}(t)}\right]
\end{aligned}
$$

The first term on the right hand side of (16) is given by

$$
\begin{aligned}
\mathbb{E}\left[\left(\Phi_{i}(t)^{\prime}\right)^{(m)} c \mathbf{1}_{\left[t_{1}>t\right]}\right] & =\left(e^{A_{i}^{\prime} t}\right)^{(m)} c \mathbb{E}\left[\mathbf{1}_{\left[t_{1}>t\right]}\right] \\
& =\left(e^{A_{i}^{\prime} t}\right)^{(m)} c S_{i}(t),
\end{aligned}
$$

where we used the fact that $\mathbb{E}\left[\mathbf{1}_{\left[t_{1}>t\right]}\right]=\mathbf{P}\left(\left[t_{1}>t\right]\right)=\Pi_{j=1}^{n_{l}} \mathbf{P}\left[h_{0}^{j}>t\right]=$ $\Pi_{j=1}^{n_{l}} S_{i, j}(t)=S_{i}(t)$. To obtain an expression for the second term on the right hand side of (16), notice first that for a function of the first jump time $G\left(t_{1}\right)$,

$$
\begin{aligned}
& \mathbb{E}\left[G\left(t_{1}\right) \mathbf{1}_{B_{l}(t)}\right]=\int_{0}^{t} \mathbb{E}\left[G(\tau) \mathbf{1}_{\left[h_{0}^{j}>\tau, j \neq l\right]} \mid \bar{h}_{0}^{l}=\tau\right] F_{i, l}(d \tau) \\
& =\int_{0}^{t} G(\tau) \Pi_{j=1, j \neq l}^{n_{l}} S_{i, j}(\tau) F_{i, l}(d \tau)=\int_{0}^{t} G(\tau) \frac{S_{i}(\tau)}{S_{i, l}(\tau)} F_{i, l}(d \tau) .
\end{aligned}
$$


Notice also that $\Phi_{i}(t)=\hat{\Phi}_{\xi_{l}(i)}\left(t-t_{1}\right)\left(J_{i, l} e^{A_{i} t_{1}}\right)$ when transition $l$ is first triggered, where $\hat{\Phi}_{\xi_{l}(i)}\left(t-t_{1}\right)$ is the transition matrix of the SHS from $t_{1}$ to $t$ starting the process at $q_{1}=\xi_{l}(i)$. Each of the terms of the summation on the right hand side of (16) can then be expressed as

$$
\begin{aligned}
& \mathbb{E}\left[\left(\Phi_{i}(t)^{\prime}\right)^{(m)} c \mathbf{1}_{B_{l}(t)}\right] \\
& =\int_{0}^{t}\left(E_{i, l}(\tau)^{\prime}\right)^{(m)} \mathbb{E}\left[\left(\hat{\Phi}_{\xi_{l}(i)}(t-\tau)^{\prime}\right)^{(m)} c\right] \frac{S_{i}(\tau)}{S_{i, l}(\tau)} F_{i, l}(d \tau) .
\end{aligned}
$$

By construction of the process $\mathbb{E}\left[\left(\hat{\Phi}_{\xi_{l}(i)}(t-\tau)^{\prime}\right)^{(m)}\right]=\mathbb{E}\left[\left(\Phi_{\xi_{l}(i)}(t-\tau)^{\prime}\right)^{(m)}\right]=$ $z_{\xi_{l}(i)}^{(m)}(t-\tau)$. Replacing (17) and (18) in (16) and noticing that $q_{0}=i \in \mathcal{Q}$ is arbitrary we obtain the desired result

$$
\begin{aligned}
z_{i}^{(m)}(t) & =\sum_{l=1}^{n_{l}} \int_{0}^{t}\left(E_{i, l}(\tau)^{\prime}\right)^{(m)} z_{\xi_{l}(i)}^{(m)}(t-\tau) \frac{S_{i}(\tau)}{S_{i, l}(\tau)} F_{i, l}(d \tau) \\
& +\left(e^{A_{i}^{\prime} t}\right)^{(m)} c S_{i}(t), \quad i \in \mathcal{Q} .
\end{aligned}
$$

Proof. (of Theorem 3) The result is a direct application of [13, Th. 1.7, Ch.3], which states that a Volterra equation taking the standard form (13) has a unique locally bounded solution given by $(15)$ provided that $\operatorname{det}\left(I-\mu^{m}(\{0\})\right) \neq$ 0 , where $\mu^{m}$ is the kernel measure of the Volterra Equation (13). In the present case $\mu^{(m)}(\{0\})$ is a $\left(n_{q} n^{m}\right) \times\left(n_{q} n^{m}\right)$ matrix with blocks $\mu_{i, j}^{(m)}(\{0\})=$ $\sum_{l=1}^{n_{l}} E_{i, l}(0)^{(m)} \mathbf{1}_{\xi_{l}(i)=j} \frac{S_{i}(0)}{S_{i, l}(0)} F_{i, l}(0), i, j \in \mathcal{Q}$. The fact that we assumed $F_{i, j}(0)=$ 0 implies that this last condition is met.

\section{Asymptotic analysis}

Even without explicitly computing the solution to the Volterra equation (13), it is possible to characterize its asymptotic behavior through a frequency-domain analysis. In this section, we restrict our attention to second-order moments and consider the expected value of a quadratic function of the systems' state $\mathbb{E}\left[x(t)^{\prime} x(t)\right]$, which is the key quantity in the stability notions that we consider here. The proofs are omitted due to space limitations, but can be found in [14].

Let $\nu$ denote the operator that transforms a matrix into a column vector $\nu(A)=\nu\left(\left[a_{1} \ldots a_{n}\right]\right)=\left[a_{1}^{\prime} \ldots a_{n}^{\prime}\right]^{\prime}$, and recall that

$$
\nu(A B C)=\left(C^{\prime} \otimes A\right) \nu(B) .
$$

Using (19) we can write the quadratic function of interest as

$$
\mathbb{E}\left[x(t)^{\prime} x(t)\right]=\mathbb{E}\left[x(t)^{\prime} \otimes x(t)^{\prime}\right] \nu\left(I_{n}\right),
$$

That is, we assume $m=2$ and $c=\nu\left(I_{n}\right)$, in (7).

To achieve the asymptotic analysis of this function, we introduce the following complex function

$$
\hat{K}(z):=\left[\begin{array}{ccc}
\hat{K}_{1,1}(z) & \ldots & \hat{K}_{1, n_{q}}(z) \\
\vdots & \ddots & \vdots \\
\hat{K}_{n_{q}, 1}(z) & \ldots & \hat{K}_{n_{q}, n_{q}}(z)
\end{array}\right],
$$


where

$$
\hat{K}_{i, j}(z):=\sum_{l=1}^{n_{l}} \int_{0}^{T_{i, l}}\left(E_{i, l}(\tau)^{\prime}\right)^{(2)} e^{-z \tau} \mathbf{1}_{\xi_{l}(i)=j} F_{i, l}(d \tau),
$$

which can be partitioned as in (1), $\hat{K}_{i, j}(z)=\hat{K}_{i, j}^{c}(z)+\hat{K}_{i, j}^{d}(z)$. Likewise, we partition $\hat{K}(z)$ as $\hat{K}(z)=\hat{K}_{c}(z)+\hat{K}_{d}(z)$, where $\hat{K}_{c}(z)$ is a matrix with blocks $\hat{K}_{i, j}^{c}(z)$, and $\hat{K}_{d}(z)$ is a matrix with blocks $\hat{K}_{i, j}^{d}(z)$. It turns out that the asymptotic stability of the Volterra equation (13) is equivalent to the following condition:

$$
\operatorname{det}(I-\hat{K}(z)) \neq 0, \quad \forall z \in \mathbb{C}: \Re[z] \geq 0,
$$

which will be instrumental in deriving the results that follow. In the sequel, we shall also provide computationally efficient methods to test (22).

To state the main result of this section we need the following technical conditions. We denote by $\mathcal{C}(R, \epsilon)$ the region in the complex plane $\mathcal{C}(R, \epsilon):=\{z$ : $|z|>R, \Re[z]>-\epsilon\}$.

(T1) $\hat{K}(-\epsilon)$ exists for some $\epsilon>0$.

(T2) $\inf _{z \in \mathcal{C}(R, \epsilon)}\left\{\left|\operatorname{det}\left(I-\hat{K}_{d}(z)\right)\right|\right\}>0$ for some $\epsilon>0, R>0$.

These conditions hold trivially when the transition distributions have bounded support $\left(T_{i, l}<\infty\right)$ and have no discrete component $\left(F_{i, l}^{d}=0\right)$. However, we shall see below that they hold under much less stringent assumptions.

We consider the following three stability notions for the SHS with renewal transitions.

Definition 4 The SHS with renewal transitions is said to be

(i) Mean Square Stable (MSS) if for any $\left(x_{0}, q_{0}\right)$,

$$
\lim _{t \rightarrow+\infty} \mathbb{E}\left[x(t)^{\prime} x(t)\right]=0,
$$

(ii) Stochastic Stable (SS) if for any $\left(x_{0}, q_{0}\right)$

$$
\int_{0}^{+\infty} \mathbb{E}\left[x(t)^{\prime} x(t)\right] d t<\infty
$$

(iii) Mean Exponentially Stable (MES) if there exists constants $c>0$ and $\alpha>0$ such that for any $\left(x_{0}, q_{0}\right)$

$$
\mathbb{E}\left[x(t)^{\prime} x(t)\right] \leq c e^{-\alpha t} x_{0}^{\prime} x_{0}, \forall_{t \geq 0} .
$$

The following is the main result of the section. We denote by $\bar{\lambda}\left(A_{i}\right)$ the real part of the eigenvalues of $A_{i}, i \in \mathcal{Q}$ with largest real part and $\bar{m}\left(A_{i}\right)$ the dimension of the largest Jordan block associated with these eigenvalues.

Theorem 5 Suppose that (T1) and (T2) hold and consider the following condition

$$
\operatorname{det}(I-\hat{K}(z)) \neq 0, \Re[z] \geq 0 .
$$

The SHS with renewal transitions is 
(i) MSS if and only if (25) holds and

$$
e^{2 \bar{\lambda}\left(A_{i}\right) t} t^{2 \bar{m}\left(A_{i}\right)} S_{i}(t) \rightarrow 0 \text { as } t \rightarrow \infty, \quad \forall_{i \in \mathcal{Q}} ;
$$

(ii) SS if and only if (25) holds and

$$
\int_{0}^{\infty} e^{2 \bar{\lambda}\left(A_{i}\right) t} t^{2 \bar{m}\left(A_{i}\right)} S_{i}(t) d t<\infty, \quad \forall_{i \in \mathcal{Q}}
$$

(iii) MES if and only if (25) holds and

$$
e^{2 \bar{\lambda}\left(A_{i}\right) t} t^{2 \bar{m}\left(A_{i}\right)} S_{i}(t) \leq c e^{-\alpha_{1} t}, \text { for } c>0, \alpha_{1}>0, \forall_{i \in \mathcal{Q}}
$$

As mentioned above, the condition (25) is a stability condition for the Volterra equation (13) and guarantees that the process sampled at the jump times converges to zero, whereas conditions (26), (27) and (28) pertain to the inter-jump behavior of the SHS with renewal transitions. In spite of not being equivalent in general, when the matrices $A_{i}, i \in \mathcal{Q}$ and the distributions $F_{i, l}, i \in \mathcal{Q}, l \in \mathcal{L}$ are such that (28) holds, then (26) and (27) automatically hold and the three notions of stability are equivalent. Note that (T1) holds if $T_{i, l}<\infty, \forall_{i \in \mathcal{Q}, l \in \mathcal{L}}$ or if all $A_{i}$ are Hurwitz, or, more generally, if for some $\lambda>\bar{\lambda}_{i}(A), \forall_{i \in \mathcal{Q}}$, we have that

$$
\int_{0}^{T_{i, l}} e^{2 \lambda s} F_{i, l}(d s)<\infty, \forall_{i \in \mathcal{Q}, l \in \mathcal{L}} .
$$

Moreover, whenever (T1) holds, the following proposition provides a simple condition to verify if (T2) holds.

Proposition 6 Assuming that (T1) holds, the condition (T2) also holds provided that

$$
\sigma\left(\hat{K}_{d}(0)\right)<1
$$

When the SHS with renewal transitions is MES, we can characterize the exponential decay constant $\alpha$ in (24) at which the expected value of the energy of the system decreases, as shown in the next Theorem.

Theorem 7 If the SHS with renewal transitions is MES, the decay constant $\alpha$ in (24) can be chosen in the interval $\alpha \in\left[0, \alpha_{\max }\right)$, where $\alpha_{\max }=\min \left\{\alpha_{1}, \alpha_{2}\right\}$, $\alpha_{1}$ is such that (28) holds, and

$$
\alpha_{2}=\left\{\begin{array}{l}
\infty, \text { if } \operatorname{det}(I-\hat{K}(a)) \neq 0, a \in(-\infty, 0), \\
\max \{a<0: \operatorname{det}(I-\hat{K}(a))=0\}, \text { otherwise. }
\end{array}\right.
$$


Suppose that the transition distributions have finite support, in which case $\alpha_{1}$ in (28) can be chosen arbitrarily large. Then this theorem tells us that the exponential decay constant of the expected value of the energy of the system is only limited by the largest real negative zero of $\operatorname{det}(I-\hat{K}(z))$, which can be obtained by performing a line search on the negative real axis.

Theorems 5 and 7 pertain to the asymptotic behavior of (13) when (25) holds. The following result characterizes the asymptotic behavior of the process when $(25)$ does not hold. Since $m=2$, we drop the index $m$ in the expressions for $z^{m}(t)$ and $h^{m}(t)$, i.e., $\zeta(t):=z^{2}(t)$ and $h(t):=h^{2}(t)$, and denote the Laplace transform of $h(t)$, by $\hat{h}(z):=\int_{0}^{\infty} h(t) e^{-z t} d t$.

Theorem 8 Suppose that (T1), (T2) and (28) hold, but (25) does not hold. Then

(i) there is a finite number $n_{z}$ of complex numbers $z_{i}$ in $\Re[z] \geq 0$ that satisfy

$$
\operatorname{det}\left(I-\hat{K}\left(z_{i}\right)\right)=0, \forall i=1, \ldots, n_{z}
$$

(ii) one number, which we label $z_{1}$, is real and verifies $\Re\left[z_{i}\right] \leq z_{1}, \forall_{i}$;

(iii) the following holds

$$
\zeta(t)=\sum_{i=1}^{n_{z}} \sum_{j=0}^{m_{i}-1} r_{i, j} t^{j-1} e^{z_{i} t}+\tilde{\zeta}(t)
$$

where $\tilde{\zeta}(t)$ tends to zero exponentially fast and the matrices $r_{i, j}$ are such that in a neighborhood of $z_{i}$,

$$
[I-\hat{K}(z)]^{-1} \hat{h}(z)=\sum_{j=0}^{m_{i}-1} r_{i, j} \frac{j !}{\left(z-z_{i}\right)^{j+1}}+y_{i}(z),
$$

where the $y_{i}(z)$ are analytic functions. Moreover, for at least one $j, r_{1, j} \neq$ 0 .

In practice, this result shows that when the SHS is not stable with respect to the stability notions considered, the expected energy $\zeta(t)$ of the SHS grows exponentially fast, according to a sum of exponential terms which are determined by the roots of $\operatorname{det}(I-\hat{K}(z))=0$ and the residues of $[I-\hat{K}(z)]^{-1} \hat{h}(z)$ in the neighborhood of these roots (singularities). The highest exponential rate is determined by the most positive real part of the roots of $\operatorname{det}\left(I-\hat{K}\left(z_{i}\right)\right)$, which turns out to be a real root, and cannot be a removable singularity.

The next result provides computationally efficient tests to verify whether or not the condition (25) holds. 
Theorem 9 The following are equivalent

(A) $\operatorname{det}(I-\hat{K}(z)) \neq 0, \Re[z] \geq 0$,

(B) $\sigma(\hat{K}(0))<1$,

(C) There exists a set of matrices $P:=\left\{P_{i}>0, i \in \mathcal{Q}\right\}$

such that for every $i \in \mathcal{Q}$

$$
L_{i}(P)-P_{i}<0
$$

where

$L_{i}(P):=$

$\sum_{j=1}^{n_{q}} \sum_{l=1}^{n_{l}} \int_{0}^{T_{i, l}} E_{i, l}(s)^{\prime} P_{j} E_{i, l}(s) \mathbf{1}_{\xi_{l}(i)=j} \frac{S_{i}(s)}{S_{i, l}(s)} F_{i, l}(d s)$,

(D) The solution to

$$
\begin{gathered}
L_{i}(P)-P_{i}=-Q_{i}, \quad i \in \mathcal{Q}, \\
\text { is unique and verifies }\left\{P_{i}>0, i \in \mathcal{Q}\right\} \text { if }\left\{Q_{i}>0, i \in \mathcal{Q}\right\} \\
\text { and }\left\{P_{i} \geq 0, i \in \mathcal{Q}\right\} \text { if }\left\{Q_{i} \geq 0, i \in \mathcal{Q}\right\} .
\end{gathered}
$$

The well-known Nyquist criterion can be used to check if (A) holds. When $\operatorname{det}(I-\hat{K}(i w)) \neq 0, \forall w \in \mathbb{R}$, the number of zeros of $\operatorname{det}(I-\hat{K}(z))$ in the closed-right half complex plane counted according to their multiplicities equals the number of times that the curve $\operatorname{det}(I-\hat{K}(i w)),-\infty<w<+\infty$ circles anticlockwise around the origin as $\omega$ goes from $\infty$ to $-\infty$. If the number of encirclements of this curve is zero then (A) holds. When $\operatorname{det}(I-\hat{K}(z))$ has zeros over the axis one needs to use the standard Nyquist criteria tricks to determine the number of zeros in the right-half plane [15]. The condition (B) is an algebraic condition, and (C) is an LMI condition since the left-hand side of (32) is an affine function of the decision variables.

\section{Special Cases}

In this section we consider the following system

$$
\begin{array}{ll}
\dot{x}(t)=A x(t), & t \neq t_{k}, \quad t \geq 0, \quad k \in \mathbb{Z}_{>0} \\
x\left(t_{k}\right)=J_{q_{k}} x\left(t_{k}^{-}\right), & t_{0}=0, x\left(t_{0}\right)=x_{0}, \quad q_{0}=\bar{q}_{0},
\end{array}
$$

where $\left\{t_{k+1}-t_{k}, k \geq 0\right\}$ are independent and identically distributed following a distribution $F$, and $q_{k} \in \mathcal{Q}=\left\{1, \ldots, n_{q}\right\}$ is a finite-dimensional Markov chain, i.e., $\mathbf{P}\left[q_{k+1}=j \mid q_{k}=i\right]=p_{i j}$. The distribution $F$ has support on an interval $[0, T]$, where $T \in \mathbb{R}_{>0} \cup\{\infty\}$. We show in this Section how one can cast (33) in the framework of SHS with renewal transitions.

If $F$ is absolutely continuous, one can directly model (33) in the framework of SHS with renewal transitions by considering: (i) $n_{q}$ discrete modes; (ii) $n_{l}=n_{q}$ reset maps taking the form (3) with $J_{i, l}=J_{l}$ and $\xi_{l}(i)=l, \forall_{q \in \mathcal{Q}, l \in \mathcal{L}}$; (iii) transition distributions given by

$$
F_{i, l}(\tau)=1-e^{-\int_{0}^{\tau} p_{i l} \lambda(a) d a},
$$


where $\lambda$ is the hazard rate of $F$, i.e., $\lambda(\tau)=\frac{\dot{F}(\tau)}{1-F(\tau)}, \tau \in[0, T), F(0)=0$. (iv) and dynamics taking the form (2) with $A_{i}=A$. In fact, noticing that $F_{i, l}(d \tau)=p_{i l} F(d \tau)$, the discrete mode sampled at transition times $q_{k}:=q\left(t_{k}\right)$ is then a Markov-chain since

$$
\begin{aligned}
\operatorname{Prob}\left[q_{k+1}=j \mid q_{k}=i\right] \\
=\mathbb{E}\left[\mathbf{1}_{q_{k+1}=j} \mid q_{k}=i\right]=\mathbb{E}\left[\mathbf{1}_{\left[h_{k}^{l}>h_{k}^{j}, l \neq j\right]} \mid q_{k}=i\right] \\
=\int_{0}^{T} \mathbb{E}\left[\mathbf{1}_{\left[h_{k}^{l}>\tau, l \neq j\right]} \mid q_{k}=i\right] F_{i, j}(d \tau) \\
=\int_{0}^{T} \Pi_{l=1, l \neq j}^{n_{l}} \mathbb{E}\left[\mathbf{1}_{\left[h_{k}^{l}>\tau\right]} \mid q_{k}=i\right] F_{i, j}(d \tau) \\
=\int_{0}^{T} \Pi_{l=1, l \neq j}^{n_{l}} S_{i, l}(\tau) F_{i, j}(d \tau) \\
=\int_{0}^{T} \Pi_{l=1, l \neq j}^{n_{l}} e^{-\int_{0}^{\tau} p_{i l} \lambda(a) d a} p_{i j} \lambda(\tau) e^{-\int_{0}^{\tau} p_{i j} \lambda(a) d a} d \tau \\
=\int_{0}^{T} p_{i j} \lambda(\tau) e^{-\int_{0}^{\tau} \lambda(a) d a} d \tau=\int_{0}^{T} p_{i j} F(d \tau)=p_{i j} .
\end{aligned}
$$

The set of Volterra equations (13) particularized to this special SHS with renewal transition take the form

$$
z_{i}^{m}(t)=\sum_{l=1}^{n_{l}} \int_{0}^{t}\left(e^{A^{\prime} \tau} J_{l}^{\prime}\right)^{(m)} z_{l}^{m}(t-\tau) p_{i l} F(d \tau)+\left(e^{A^{\prime} t}\right)^{(m)} S(t)
$$

where $S(t):=1-F(t)$. The results of the previous sections can then be applied to $(33)$.

The approach followed so far, is suggested in [5] to extend the class of SHS to the case where the next state is chosen according to a distribution, which is the case in (33). However, it requires $F$ to be absolutely continuous, due to Assumption 1. One can drop this restriction by considering a more natural execution of a sample path of (33) than the one provided in Section 2 for a sample path of a SHS with renewal transitions. Consider the following description of a sample-path of (33).

1. Set $k=0, t_{0}=0,\left(q\left(t_{k}\right), x\left(t_{k}\right)\right)=\left(q_{0}, x_{0}\right)$.

2. Obtain $h_{k}$ as a realization of a random variable distributed according to $F(\tau)$ and set the next transition time to $t_{k+1}=t_{k}+h_{k}$. The state of (33) in the interval $t \in\left[t_{k}, t_{k+1}\right)$ is given by $(q(t), x(t))=\left(q\left(t_{k}\right), e^{A t} x\left(t_{k}\right)\right)$.

3. Obtain $q_{k+1}$ as discrete random variable distributed according to $\mathbf{P}\left[q_{k+1}=\right.$ $j]=p_{q\left(t_{k}^{-}\right) j}, j \in \mathcal{Q}$. Update the state according to

$$
\left(q\left(t_{k+1}\right), x\left(t_{k+1}\right)\right)=\left(q_{k+1}, J_{q_{k+1}} x\left(t_{k+1}^{-}\right)\right) .
$$

Set $k=k+1$ and repeat the construction from the step 2 .

The next Theorem states that with this interpretation of a sample path of (33), the set of Volterra equations (35) hold, even in the case where $F$ is not 
absolutely continuous. Analogously to (9), let $z_{i}^{m}(t):=\mathbb{E}\left[\left(\Phi_{i}(t)\right)^{m} c\right], i \in \mathcal{Q}$, where $\Phi_{i}(t)$ is the transition matrix of (33) with initial state $q_{0}=i$, i.e.,

$$
\Phi_{i}(t)=e^{A\left(t-t_{r}\right)} J_{q\left(t_{r}\right)} \ldots e^{A h_{1}} J_{q_{1}} e^{A h_{0}}, \mathbf{P}\left[q_{1}=l\right]=p_{i l}, l \in \mathcal{L}
$$

Theorem 10 The following holds for the system (33) with the sample-description given in this section

$$
z_{i}^{m}(t)=\sum_{l=1}^{n_{l}} \int_{0}^{t}\left(e^{A^{\prime} \tau} J_{l}^{\prime}\right)^{(m)} z_{l}^{m}(t-\tau) p_{i l} F(d \tau)+\left(e^{A^{\prime} t}\right)^{(m)} c S(t)
$$

Proof. Consider an initial condition $q_{0}=i, i \in \mathcal{Q}$ and a given time $t$ and partition the probability space $\boldsymbol{\Omega}$ into $\boldsymbol{\Omega}=\left[t_{1} \leq t\right] \cup\left[t_{1}>t\right]$. We can further partition $\left[t_{1} \leq t\right]$ into $\cup_{l=1}^{n_{l}}\left[t_{1} \leq t\right] \wedge\left[q_{1}=l\right]$. Notice that, since the initial state is $q_{0}=i, \mathbf{P}\left[q_{1}=l\right]=p_{i l}$. Using this partition we can write

$$
\begin{aligned}
\mathbb{E}\left[\left(\Phi_{i}(t)^{\prime}\right)^{(m)} c\right] & =\mathbb{E}\left[\left(\Phi_{i}(t)^{\prime}\right)^{(m)} c \mathbf{1}_{\left[t_{1}>t\right]}\right] \\
& +\sum_{l=1}^{n_{l}} \mathbb{E}\left[\left(\Phi_{i}(t)^{\prime}\right)^{(m)} c \mathbf{1}_{\left[t_{1} \leq t\right] \wedge\left[q_{1}=l\right]}\right]
\end{aligned}
$$

The first term on the right hand side of (36) is given by

$$
\begin{aligned}
\mathbb{E}\left[\left(\Phi_{i}(t)^{\prime}\right)^{(m)} c \mathbf{1}_{\left[t_{1}>t\right]}\right] & =\left(e^{A^{\prime} t}\right)^{(m)} c \mathbb{E}\left[\mathbf{1}_{\left[t_{1}>t\right]}\right] \\
& =\left(e^{A^{\prime} t}\right)^{(m)} c S(t),
\end{aligned}
$$

To obtain an expression for the second term on the right hand side of (36), note that $\Phi_{i}(t)=\hat{\Phi}_{l}\left(t-t_{1}\right)\left(J_{l} e^{A t_{1}}\right)$, where $\hat{\Phi}_{l}(t-s)$ is the transition matrix of the SHS from $s=t_{1}$ to $t$ starting the process at $q_{1}=l$. Each of the terms of the summation on the right hand side of (36) can then be expressed as

$$
\begin{aligned}
\mathbb{E}\left[\left(\Phi_{i}(t)^{m}\right)^{\prime} c \mathbf{1}_{\left[t_{1} \leq t\right] \wedge\left[q_{1}=l\right]}\right] & \\
& =\int_{0}^{t}\left(e^{A^{\prime} \tau} J_{l}^{\prime}\right)^{(m)} \mathbb{E}\left[\left(\hat{\Phi}_{l}(t-\tau)^{(m)}\right)^{\prime} c\right] p_{i l} F(d \tau) .
\end{aligned}
$$

By construction of the process $\mathbb{E}\left[\hat{\Phi}_{l}(t-s)\right]=\mathbb{E}\left[\Phi_{l}(t-s)\right]=z_{l}^{(m)}(t-s)$. Replacing (37) and (38) in (36), using the notation (9) and noticing that $q_{0}=i \in \mathcal{Q}$ is arbitrary we obtain the desired result

$$
z_{i}^{m}(t)=\sum_{l=1}^{n_{l}} \int_{0}^{t}\left(e^{A^{\prime} \tau} J_{l}^{\prime}\right)^{(m)} z_{l}^{m}(t-\tau) p_{i l} F(d \tau)+\left(e^{A^{\prime} t}\right)^{(m)} c S(t)
$$

The results of the previous sections can then be specialized to the system (33), even in the case where $F$ is not absolutely continuous, since they rely only on the Volterra equation (35), and not on the particular interpretation given to a sample path of (33). In particular we state the following Theorem, which combines Theorem 5 and 9 . Let

$$
\hat{K}_{e}(z):=\left[\begin{array}{ccc}
\hat{K}_{e 1,1}(z) & \ldots & \hat{K}_{e 1, n_{q}}(z) \\
\vdots & \ddots & \vdots \\
\hat{K}_{e n_{q}, 1}(z) & \ldots & \hat{K}_{e n_{q}, n_{q}}(z)
\end{array}\right],
$$


where $\hat{K}_{i, l}(z):=p_{i l} \int_{0}^{T}\left(J_{l} e^{A s}\right)^{\prime} \otimes\left(J_{l} e^{A s}\right)^{\prime} e^{-z s} F(d s)$ and consider the two technical conditions,

(T1e) $\hat{K}_{e}(-\epsilon)$ exists for some $\epsilon>0$.

(T2e) $\inf _{z \in \mathcal{C}(R, \epsilon)}\left\{\left|\operatorname{det}\left(I-\hat{K}_{e d}(z)\right)\right|\right\}>0$ for some $\epsilon, R>0$.

where $\left.\hat{K}_{e d}(z)\right)$ is the discrete part of, $\hat{K}_{e}(z)=\hat{K}_{e c}(z)+\hat{K}_{e d}(z)$ partitioned as in (1). We denote by $\bar{\lambda}(A)$ the real part of the eigenvalues of $A$ with largest real part and $\bar{m}(A)$ the dimension of the largest Jordan block associated with these eigenvalues.

Theorem 11 The following are equivalent

(A) $\operatorname{det}\left(I-\hat{K}_{e}(z)\right) \neq 0, \Re[z] \geq 0$,

(B) $\quad \sigma\left(\hat{K}_{e}(0)\right)<1$,

(C) There exists a set of matrices $P:=\left\{P_{i}>0, i \in \mathcal{Q}\right\}$

such that for every $i \in \mathcal{Q}$

$L_{e i}(P)-P_{i}<0$,

where $L_{e i}(P):=\sum_{l=1}^{n_{q}} p_{i l} \int_{0}^{T}\left(J_{l} e^{A s}\right)^{\prime} P_{j} J_{l} e^{A s} F(d s)$,

(D) The solution to

$$
L_{e i}(P)-P_{i}=-Q_{i}, \quad i \in \mathcal{Q},
$$

is unique and verifies $\left\{P_{i}>0, i \in \mathcal{Q}\right\}$ if $\left\{Q_{i}>0, i \in \mathcal{Q}\right\}$

$\operatorname{and}\left\{P_{i} \geq 0, i \in \mathcal{Q}\right\}$ if $\left\{Q_{i} \geq 0, i \in \mathcal{Q}\right\}$.

Furthermore, assuming (T1e) and (T2e), the system (33) is

(i) MSS if and only if any of (A)-(D) holds and

$$
e^{2 \bar{\lambda}(A) t} t^{2 \bar{m}(A)} S(t) \rightarrow 0 \text { as } t \rightarrow \infty ;
$$

(ii) SS if and only if any of (A)-(D) holds and

$$
\int_{0}^{\infty} e^{2 \bar{\lambda}(A) t} t^{2 \bar{m}(A)} S(t) d t<\infty ;
$$

(iii) MES if and only if any of (A)-(D) holds and

$$
e^{2 \bar{\lambda}(A) t} t^{2 \bar{m}(A)} S(t) \leq c e^{-\alpha_{1} t} \text { for some } c>0, \alpha_{1}>0 .
$$

A special case of (33) is the following system

$$
\begin{array}{ll}
\dot{x}(t)=A x(t), & t \neq t_{k}, \quad t \geq 0, \quad k \in \mathbb{Z}_{>0} \\
x\left(t_{k}\right)=J_{k}^{\omega_{k}} x\left(t_{k}^{-}\right), & t_{0}=0, x\left(t_{0}\right)=x_{0},
\end{array}
$$

where $\left\{\omega_{k} \in\left\{1, \ldots, n_{J}\right\}, k \geq 0\right\}$ are independent and identically distributed random variables, $\bar{p}_{i}:=P\left[w_{k}=i\right], i \in\left\{1, \ldots, n_{\omega}\right\}, \forall_{k}$, and the dependence 
of $J_{k}^{\omega_{k}}$ on $k$ is $n_{J}$ periodic, i.e, $J_{k}^{\omega_{k}}=J_{k+n_{J}}^{\omega_{k}}, \forall_{k}$. We can cast this system in the framework of (33) by considering $n_{q}=n_{\omega} \times n_{J}$ states of the Markov-Chain labeled, for example, according to the correspondence $\left(\bmod \left(k, n_{J}\right)+1=\kappa, w_{k}=\right.$ $l) \rightarrow q_{k}=l+(\kappa-1) n_{\omega}, l \in\left\{1, \ldots, n_{\omega}\right\}, \kappa \in\left\{1, \ldots, n_{J}\right\}$, where $\bmod \left(k, n_{J}\right)$ is the remainder after division of $k$ by $n_{J}$, and easily obtain $p_{i j}$ as

$$
\left[p_{i j}\right]=\left[\begin{array}{ccccc}
0 & \bar{P} & 0 & \ldots & 0 \\
0 & 0 & \bar{P} & \ldots & 0 \\
\vdots & \vdots & \vdots & \ddots & \vdots \\
0 & 0 & \ldots & 0 & \bar{P} \\
\bar{P} & 0 & \ldots & 0 & 0
\end{array}\right]
$$

where $\bar{P} \in \mathbb{R}^{n_{\omega} \times n_{\omega}}=\bar{p} 1_{n_{\omega}}^{\prime}, \bar{p}=\left[\begin{array}{llll}\bar{p}_{1} & \bar{p}_{2} & \ldots & \bar{p}_{n}\end{array}\right]$ and $1_{n_{\omega}}$ is a vector of $n_{\omega}$ ones. We can then also state the results for the system given in the previous sections and specialized to (33). However there is redundancy in this procedure. We illustrate this redundancy, stating that stability of (43) is equivalent to a lower dimension LMI, than the one we would obtain by doing this procedure.

Proposition 12 Suppose that (42) holds. Then, the system (43) is MSS, SS and $M E S$ if and only if

$$
\begin{gathered}
\exists_{\left\{P_{\kappa}>0\right\}}: \sum_{i=1}^{n_{\omega}} \bar{p}_{i} \int_{0}^{T}\left(J_{[\kappa+1]}^{i} e^{A \tau}\right)^{\prime} P_{[\kappa+1]} J_{[\kappa+1]}^{i} e^{A \tau} F(d \tau)-P_{\kappa}, \\
\kappa \in\left\{1, \ldots, n_{J}\right\},
\end{gathered}
$$

where $[\kappa+1]:=1$ if $\kappa=n_{J}$, and $[\kappa]:=\kappa$ otherwise.

Notice that by properly relabeling the $P_{\kappa}(46)$ is equivalent to:

$$
\begin{array}{r}
\exists_{\left\{P_{\kappa}>0\right\}}: \sum_{i=1}^{n_{\omega}} \bar{p}_{i} \int_{0}^{T}\left(J_{\kappa}^{i} e^{A \tau}\right)^{\prime} P_{[\kappa+1]} J_{\kappa}^{i} e^{A \tau} F(d \tau)-P_{\kappa}, \\
\kappa \in\left\{1, \ldots, n_{J}\right\},
\end{array}
$$

\section{Proof.}

Taking into account the procedure given above to reduce the system (43) to (33), we can apply Theorem 11 and conclude that (43) is MSS, SS and MES if and only if (39) holds where the $p_{i j}$ are given by (44). Due to the block structure of (44), we can write (39) as

$$
\begin{aligned}
& \exists_{X_{i+(\kappa-1) n_{\omega}}>0}: \hat{L}_{e \kappa}\left(\hat{X}_{[\kappa+1]}\right)-X_{i+(\kappa-1) n_{\omega}}<0, \\
& \forall i \in\left\{1, \ldots, n_{\omega}\right\}, \forall \kappa \in\left\{1, \ldots, n_{J}\right\}
\end{aligned}
$$

where $\hat{X}_{[\kappa+1]}:=\left(X_{([\kappa+1]-1) n_{\omega}+1}, \ldots, X_{[\kappa+1] n_{\omega}}\right)$ and

$$
\hat{L}_{e}\left(\hat{X}_{[\kappa+1]}\right)=\sum_{i=1}^{n_{\omega}} \bar{p}_{i} \int_{0}^{T}\left(J_{[\kappa+1]}^{i} e^{A \tau}\right)^{\prime} X_{([\kappa+1]-1) n_{\omega}+i} J_{[\kappa+1]}^{i} e^{A \tau} F(d \tau)
$$

If there exists a solution $\left\{P_{\kappa}, \kappa \in\left\{1, \ldots, n_{J}\right\}\right\}$ to $(45)$ then

$$
\hat{X}_{\kappa}=\left(P_{\kappa}, \ldots, P_{\kappa}\right), \kappa \in\left\{1, \ldots, n_{J}\right\}
$$


is a solution to (47).

If there exists a solution $\left\{X_{\iota}, 1 \leq \iota \leq n_{\omega} n_{J}\right\}$ to (47), then

$$
P_{\kappa}=\frac{1}{n_{\omega}}\left(\sum_{l=1}^{n_{\omega}} X_{([k+1]-1) n_{\omega}+l}\right)
$$

is a solution to (45). We can conclude this by summing (47) for fixed $\kappa$ and $i$ running from 1 to $n_{\omega}$ and dividing by $n_{\omega}$.

\section{Application to Networked Control}

We consider the following simplified version of the networked control set-up we considered in [10]. Suppose that we wish to control a linear plant

$$
\dot{x}_{P}(t)=A_{P} x_{P}(t)+B_{P} \hat{u}(t),
$$

where the actuation is held constant $\hat{u}(t)=\hat{u}\left(s_{\kappa}\right), t \in\left[s_{\kappa}, s_{\kappa+1}\right)$ between actuation updates, denoted by $\left\{s_{\kappa}, \kappa \geq 0\right\}$. We assume that the sensors provide full state measurements that are available on demand to a digital controller. The controller computes a state-feedback control law, as follows

$$
u_{\kappa}=K_{C} x_{P}\left(r_{\kappa}\right) \text {, }
$$

where $\left\{r_{\kappa}, \kappa \geq 0\right\}$ are the times at which the controller demands a sensor sample. The controller gains are assumed to be stabilizing in the ideal case where $\hat{u}(t)=$ $K_{C} x_{P}(t)$, i.e., $A_{P}+B_{P} K_{C}$ is Hurwitz.

The controller and the actuator are connected through a network possibly shared by other users. The network is assumed to provide the following service. Whenever a user has data to transmit it waits a random amount of time until it gets to transmit its message through the network. The waiting times are independent and identically distributed. As noted in [10], this network model is reasonable in scenarios in which nodes attempt to do periodic transmissions of data, but these regular transmissions may be perturbed by the medium access protocol. For example, nodes using CSMA for medium access, may be forced to back-off for a typically random amount of time until the network becomes available.

The digital controller proceeds as follows. It samples the sensor data, computes the control law and initiates the transmission process, i.e, waits for a random amount of time until it gains access to the network and transmits the control law to the plant's actuators. Hereafter we refer to the distribution of these independent waiting times as the network distribution, and denote it by $F(\tau)$. After transmitting the control law data, it waits a given time $T_{s}$ which would correspond to a sampling period if the delay in the transmission process were zero, after which it samples the sensor data and repeats the process.

We consider two different cases:

Case I: After waiting to obtain network access, the controller (re)samples the sensor, computes the control law and transmits it, i.e., the most recent data. This case is the most reasonable when transmitting dynamic data. Assuming that the transmission delays are negligible, the sampling of the sensors occurs at 
the same times at which data arrives to the plant's actuators and the actuation is updated, and therefore $r_{k}=s_{k}:=t_{k}, \forall_{k}$. Defining $x:=\left(x_{P}, \hat{u}\right)$, we have

$$
\begin{gathered}
\dot{x}=A x, \quad A=\left[\begin{array}{cc}
A_{P} & B_{P} \\
0 & 0
\end{array}\right], \\
x\left(t_{k}\right)=J x\left(t_{k}^{-}\right), \quad J=\left[\begin{array}{cc}
I & 0 \\
K_{C} & 0
\end{array}\right] .
\end{gathered}
$$

Since in the interval $t_{k+1}-t_{k}$ the controller waits a fixed time $T_{s}$ and a random amount of time following a distribution $F(s)$, the intervals $\left\{t_{k+1}-t_{k}, k \geq 0\right\}$ are independent and identically distributed according to

$$
\tilde{F}(\tau)=\left\{\begin{array}{l}
F\left(\tau-T_{s}\right) \text { if } \tau \geq T_{s} \\
0, \quad t \in\left[0, T_{s}\right) .
\end{array}\right.
$$

Note that the system (49) is a special case of a SHS with a single state and a single reset map.

Case II: After waiting to obtain network access, the controller transmits the data collected at the time it initially tried to transmit data. This case is more realistic than the first one since the controller typically sends the sensor data to the network adapter and does not have the option to update this data at the transmission time. We model this by a SHS with the following two discretemodes $\left(n_{q}=2\right)$,

- State $i=1$ - The controller waits the random time to gain access to the network and transmit the actuation data.

- State $i=2$ - The controller waits $T_{s}$ seconds, where $T_{s}$ would correspond to a sampling period if the network delay (i.e., the waiting time in state $i=1$ ) was zero.

Let $v(t):=u_{\kappa}, t \in\left[r_{\kappa}, r_{\kappa+1}\right)$ be a variable that holds the last computed control value, and let $x:=\left(x_{P}, \hat{u}, v\right)$. The transitions between the two discrete modes can be modeled by a single transition function $\left(n_{l}=1\right)$ taking the form (3) which is specified as follows. When in state 2 the SHS transits to state $1\left(\xi_{1}(2)=1\right)$ at sampling times $r_{\kappa}$. The state jump models the update of the variable $v\left(r_{\kappa}\right)=u_{\kappa}$ that holds the last computed control value

$$
x\left(r_{\kappa}\right)=J_{2,1} x\left(r_{\kappa}^{-}\right), \quad J_{2,1}=\left[\begin{array}{ccc}
I & 0 & 0 \\
0 & I & 0 \\
K_{C} & 0 & 0
\end{array}\right] .
$$

When in state 1 the SHS transits to state $2\left(\xi_{1}(1)=2\right)$ at actuation update times $s_{\kappa}$. The state jump models the actuation update $\hat{u}\left(s_{\kappa}\right)=v\left(s_{\kappa}^{-}\right)=v\left(r_{\kappa}\right)$

$$
x\left(s_{\kappa}\right)=J_{1,1} x\left(s_{\kappa}^{-}\right), \quad J_{1,1}=\left[\begin{array}{ccc}
I & 0 & 0 \\
0 & 0 & I \\
0 & 0 & I
\end{array}\right] .
$$

Note that, the times at which transitions occur are $t_{k}:=\left\{r_{\kappa}\right\} \cup\left\{s_{\kappa}\right\}$ and the following hold $s_{\kappa}<r_{\kappa+1}<s_{\kappa+1}, r_{\kappa}=s_{\kappa}+T_{s}, \forall_{\kappa}$. The transition distributions are given by 
- $F_{1,1}(\tau)=F(\tau)$ where $F(\tau)$ is the network distribution.

- $F_{2,1}(\tau)=\delta\left(\tau-T_{s}\right)$ is a discrete distribution that places mass $w_{i}=1$ at $T_{s}$.

In both discrete-time states, the continuous-time dynamics is described by $\dot{x}=A_{i} x, i \in\{1,2\}, A_{1}=A_{2}=A$ where

$$
A=\left[\begin{array}{ccc}
A_{P} & B_{P} & 0 \\
0 & 0 & 0 \\
0 & 0 & 0
\end{array}\right] .
$$

\subsection{Numerical Example}

Suppose that the plant (48) is described by

$$
A_{P}=\left[\begin{array}{ll}
0 & 1 \\
1 & 0
\end{array}\right], \quad B_{P}=\left[\begin{array}{l}
0 \\
1
\end{array}\right]
$$

which by properly scaling the state and input can be viewed as a linearized model of a damp-free inverted pendulum. Moreover suppose that the network distribution $F(\tau)$ is uniform with support on the interval $[0, T]$, and fix $T_{s}=$ $0.1 s$. A continuous-time state feedback controller is synthesized assuming that controller and plant's actuators are directly connected, i.e., network-free. It is an LQR controller given by

$$
\hat{u}(t)=K_{C} x(t), \quad K_{C}=-\left[\begin{array}{ll}
1+\sqrt{2} & 1+\sqrt{2}
\end{array}\right],
$$

which is the solution to the problem

$$
\min _{\hat{u}(t)} \int_{0}^{\infty}\left[x_{P}(t)^{\prime} x_{P}(t)+\hat{u}(t)^{2}\right] d t,
$$

yielding $\lambda_{i}\left(A_{P}+B_{P} K_{C}\right)=\{-1,-\sqrt{2}\}$. We wish to assert the lost of stability and performance in the closed-loop when instead of the networked-free case, the controller sends its data to the actuator through a shared network, in the two scenarios considered above labeled as Cases I and II. To this effect we define the quantity

$$
y(t)=x_{P}(t)^{\prime} x_{P}(t)+\hat{u}(t)^{2},
$$

which can be written as $y(t)=x^{\prime} P x$, where in the network-free case $P=$ $I_{2}+K_{C}^{\prime} K_{C}$, and $x=x_{P}$; in case I, $P=I_{3}$ and $x=\left(x_{P}, \hat{u}\right)$; and in case II, $P=\operatorname{diag}\left(I_{2}, 1,0\right)$, and $x=\left(x_{P}, \hat{u}, v\right)$. Note that, in the network-free case, $y(t)$ is the quantity whose integral is minimized for control synthesis and $y(t)$ decreases exponentially fast at a rate $\alpha=2$, since the dominant closed-loop eigenvalue equals $\lambda_{i}\left(A_{C}+B_{K}\right)=-1$. Note also that in cases I and II, $\mathbb{E}[y(t)]$ tends to zero is equivalent to MSS, which is equivalent to SS and MES since the transition distributions have finite support ( $T$ and $T_{s}$ are finite). The conditions of Theorems 5, 9 can be used to assert if for a given $T$, the closed-loop in cases I and II is MSS. Moreover, when the closed-loop is MSS, we can determine the exponential decay constant of $\mathbb{E}[y(t)]$ by Theorem 7 . The results are summarized in Table 1. 
Table 1: Stability conditions and exponential decay rates $E[y(t)] \leq c e^{-\alpha t}$, for various values of the network distribution support in two networked control scenarios

\begin{tabular}{|c|c|c|c|c|c|c|}
\hline$T$ & 0.1 & 0.2 & 0.3 & 0.4 & 0.5 & $>0.521$ \\
\hline$\alpha$ & 2.000 & 2.000 & 2.000 & 0.849 & 0.118 & NOT MSS \\
\hline
\end{tabular}

(a) Case I

\begin{tabular}{|c|c|c|c|c|c|c|}
\hline$T$ & 0.4 & 0.6 & 0.8 & 1.0 & 1.2 & $>1.211$ \\
\hline$\alpha$ & 2.000 & 2.000 & 1.969 & 0.477 & $7.63 \times 10^{-5}$ & NOT MSS \\
\hline
\end{tabular}

(b) Case II

The fact that closed-loop stability in preserved for larger values of $T$ in Case I, confirms what one would expect intuitively, i.e, Case I is more appropriate when transmitting dynamic data, since the most recent sampling information is sent through the network.

Using the state moment expressions provided by Theorems 2 and 3, we can perform a more detailed analysis by plotting the moments of $y(t)$. These can be expressed in terms of the moments of the state. For example the two first moments take the form

$$
\begin{aligned}
& \mathbb{E}[y(t)]=\mathbb{E}\left[x(t)^{\prime} P x(t)\right]=\mathbb{E}\left[x(t)^{\prime(2)}\right] \nu(P), \\
& \mathbb{E}\left[y(t)^{2}\right]=\mathbb{E}\left[\left(x(t)^{\prime} P x(t)\right)^{2}\right]=\mathbb{E}\left[\left(x(t)^{\prime}\right)^{(4)}\right](\nu(P) \otimes \nu(P)) .
\end{aligned}
$$

In Figure 1, we plot $\mathbb{E}[y(t)]$ and $\mathbb{E}[y(t)] \pm 2 \mathbb{E}\left[(y(t)-\mathbb{E}[y(t)])^{2}\right]^{1 / 2}$ for a network distribution support $T=0.4$. Note that from the Chebyshev inequality

$$
\mathbf{P}[|y(t)-\mathbb{E}[y(t)]|>a(t)] \leq \frac{\mathbb{E}\left[(y(t)-\mathbb{E}[y(t)])^{2}\right]}{a(t)^{2}},
$$

one can guarantee that for a fixed $t, y(t)$ lies between the curves $\mathbb{E}[y(t)] \pm$ $a(t), a(t)=2 \mathbb{E}\left[(y(t)-\mathbb{E}[y(t)])^{2}\right]^{1 / 2}$ with a probability greater than $\frac{3}{4}$. The numerical method used to compute the solution of the Volterra-equation is based on a trapezoidal integration method [12]. In case I, the expected value of the quadratic state function $y(t)$ tends to zero much faster, and with a much smaller variance than in case II , confirming once again that case I is more appropriate when transmitting dynamic data.

\section{Conclusions and Future Work}

We proposed an approach based on Volterra renewal-type equations to analyze SHSs for which the lengths of times that the system stays in each mode are independent random variables with given distributions. We showed that any statistical $m$-th order moment of the state can be computed using this approach, and provided a number of results characterizing the asymptotic behavior of a second-order moment of the system. An interesting topic for future work is to extend the asymptotic analysis, to any $m$-th order moment of the state. The applicability of the results was shown in a networked control problem, where SHSs allowed us to consider network delays, which was not possible in a 


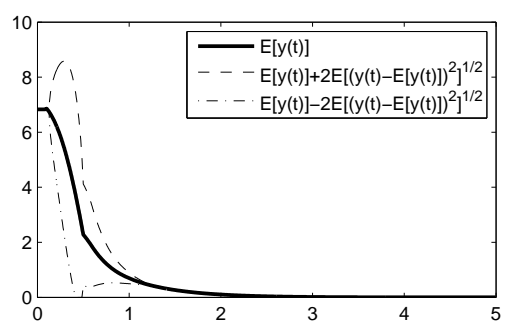

(a) Case I

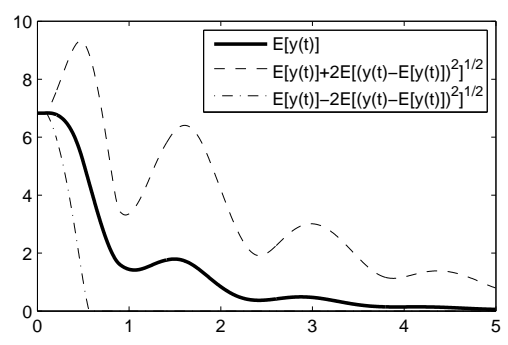

(b) Case II

Figure 1: Plot of $\mathbb{E}[y(t)]$, where $y(t)$ is quadratically state dependent. For a fixed $t, \mathbb{E}[y(t)]$ lies between the dashed curves with probability $>\frac{3}{4}$.

previous work [10]. Due to the large number of problems that fit the stochastic hybrid systems framework, finding more applications where the results can be applied is a topic for future work.

\section{A Proofs}

\section{A.1 Preliminaries}

Let $S_{+}^{n}\left(\mathbb{R}, n_{q}\right)$ denote the space of $n_{q}$ positive definite $n \times n$ matrices, i.e., $X \in S_{+}^{n}\left(\mathbb{R}, n_{q}\right)$ if $X=\left(X_{1}, \ldots, X_{n_{q}}\right), X_{i} \geq 0, i \in \mathcal{Q}$. We can interpret $\left(X_{1}, \ldots, X_{n_{q}}\right)$ as the cartesian product of $n_{q}$ matrices but it is useful to interpret $\left(X_{1}, \ldots, X_{n_{q}}\right)$ following our notation, i.e., $\left(X_{1}, \ldots, X_{n_{q}}\right)=\left[X_{1}^{\prime} \ldots X_{n_{q}}^{\prime}\right]^{\prime}$ is a matrix in $\mathbb{R}^{n_{q}(n \times n)}$, or by other words naturally identify the two spaces. The sum, product by a scalar, $\geq$ and $\nu($.$) operations are naturally defined,$ i.e, $\left.\nu\left(\left(X_{1}, \ldots, X_{n_{q}}\right)\right)=\left(\nu\left(X_{1}\right), \ldots, \nu\left(X_{n_{q}}\right)\right)\right)$ and if $Y=\left(Y_{1}, \ldots, Y_{n_{q}}\right)$ and $Z=\left(Z_{1}, \ldots, Z_{n_{q}}\right)$ then $Y+Z=\left(Y_{1}+Z_{1}, \ldots, Y_{n_{q}}+Z_{n_{q}}\right), \alpha Y=\left(\alpha Y_{1}, \ldots, \alpha Y_{n_{q}}\right)$ $Y \geq Z$ if and only if $Y_{i} \geq Z_{i}$. We define $\nu^{-1}$ as $\nu^{-1}(\nu(Y))=Y$, for $Y=$ $S_{+}^{n}\left(\mathbb{C}, n_{q}\right)$.

It will be useful to define $Z(t):=\left(Z_{1}(t), \ldots, Z_{n_{q}}(t)\right)$, where

$$
Z_{i}(t):=\mathbb{E}\left[\Phi_{i}(t)^{\prime} \Phi_{i}(t)\right], \quad i \in \mathcal{Q}
$$

Note that, using (19) we have $\nu\left(Z_{i}(t)\right)=\mathbb{E}\left[\Phi_{i}(t)^{(2)} \nu(I)\right]=z_{i}^{2}(t)=\zeta_{i}(t)$. Note also that, for the case of interest that we consider in this section particu- 
larized to $m=2$, the Volterra equation (13), can be written as

$$
\zeta(t)=K_{z}(\zeta(t))+h(t), \quad t \geq 0
$$

where $K_{z}:=K_{2}$ or in the following useful matrix form

$$
Z(t)=K(Z(t))+H(t), \quad t \geq 0
$$

where

$$
\begin{gathered}
K(Z(t)):=\left(K_{1}(Z(t)), \ldots, K_{n_{q}}(Z(t)),\right. \\
K_{i}(Z(t)):=\sum_{l=1}^{n_{l}} \int_{0}^{t} E_{i, l}(\tau)^{\prime} Z_{\xi_{l}(i)}(t-\tau) E_{i, l}(\tau) \frac{S_{i}(\tau)}{S_{i, l}(\tau)} F_{i, l}(d \tau), \quad i \in \mathcal{Q},
\end{gathered}
$$

and $H(t)=\left(H_{1}(t), \ldots, H_{n_{q}}(t)\right)$, is such that $\nu(H(t))=h(t)$, i.e.,

$$
H_{i}(t):=e^{A_{i}^{\prime} t} e^{A_{i} t}, i \in \mathcal{Q} .
$$

One can check that (52) holds by applying $\nu($.$) on both sides of (52) and$ using (19), which leads to (13) when $m=2$. Analogously to (15) we have that the unique solution to (52) is given by

$$
Z(t)=\sum_{j=1}^{\infty} K^{j}(H(t))+H(t), \quad t \geq 0 .
$$

We start by stating and proving three important Theorems (13, 14 and 15), which play a fundamental role in establishing the results that we prove in this subsection, that is, Theorems 5, 7, 8, 9 and Proposition 6 . The first two establish important properties for $\hat{K}(z)$. Theorem 15 is used to extend an instability result for Volterra equations [13, Th. 2.4 and $2.5, \mathrm{Ch} .7]$ to the critical case, where the characteristic equation has zeros on the imaginary axis, exploring the structure of the Volterra equation at hand.

\section{A.2 Preliminary Theorems}

Theorem 13 Suppose that $\hat{K}(a)$ exists for some $a \in \mathbb{R}$. Then for any $z$ : $\Re[z] \geq a$, we have $\sigma(\hat{K}(z)) \leq \sigma(\hat{K}(a))$.

Theorem 14 Suppose that $\hat{K}(a)$ exists for some $a \in \mathbb{R}$ and $\sigma(\hat{K}(a)) \geq 1$. Then there exists $a_{1} \geq a$ with the following properties: (i) $\operatorname{det}\left(I-\hat{K}\left(a_{1}\right)\right)=0$, (ii) there exists $X \geq 0$ such that $\hat{K}\left(a_{1}\right) \nu(X)=\nu(X)$, and (iii) if $z$ verifies $\operatorname{det}(I-\hat{K}(z))=0$ then $\Re[z] \leq a_{1}$.

Theorem 15 Denote by $\hat{K}_{\delta}(z), H_{\delta}(t), Z_{\delta}(t)$ the analogous functions to $\hat{K}(z)$, $H(t), Z(t)$, obtained by substituting $A_{i}$ by $A_{\delta i}=A_{i}+\frac{\delta}{2}, \delta \in \mathbb{R}, i \in \mathcal{Q}$, in (21), (54), and (50), respectively. Then, $\hat{K}_{\delta}(z)=\hat{K}(z-\delta), H_{\delta}(t)=H(t) e^{\delta t}$, and $Z_{\delta}(t)=Z(t) e^{\delta t}$. 
Proof. (of Theorem 15) The facts that $\hat{K}_{\delta}(z)=\hat{K}(z-\delta)$ and $H_{\delta}(t)=H(t) e^{\delta t}$ follow simply by replacing $A_{i}$ by $A_{i \delta}, i \in \mathcal{Q}$ in (21), (54), respectively. Let $K_{\delta}$ denote the analogous operator to $K$, obtained by substituting $A_{i}$ by $A_{\delta i}$, $i \in \mathcal{Q}$ in (53). Since by Theorems 2 and $3, Z_{\delta}(t)$ is the unique solution to $Z_{\delta}(t)=K_{\delta}\left(Z_{\delta}(t)\right)+H_{\delta}(t)$, if suffices to verify that $Z_{\delta}(t)=Z(t) e^{\delta t}$ satisfies this equation. To this effect notice that

$$
\begin{aligned}
K_{\delta i}\left(Z_{\delta}(t)\right) & =\sum_{l=1}^{n_{l}} \int_{0}^{t}\left(J_{i, l} e^{\left(A+\frac{\delta}{2} I\right) s}\right)^{\prime} Z_{\delta \xi_{l}(i)}(t-s) J_{i, l} e^{\left(A+\frac{\delta}{2} I\right) s} \frac{S_{i}(s)}{S_{i, l}(s)} F_{i, l}(d s), \\
& =\sum_{l=1}^{n_{l}} \int_{0}^{t}\left(J_{i, l} e^{A s}\right)^{\prime} Z_{\xi_{l}(i)}(t-s) e^{\delta(t-s)} J_{i, l} e^{A s} e^{\delta s} \frac{S_{i}(s)}{S_{i, l}(s)} F_{i, l}(d s), \\
& =e^{\delta t} K_{i}(Z(t)), \quad i \in \mathcal{Q},
\end{aligned}
$$

from which $Z_{\delta}(t)=e^{\delta t}(K(Z(t))+H(t))=e^{\delta t} Z(t)$.

To prove Theorems 13 and 14 we need some preliminary results. The following Theorem is taken from [16, Th. 9.1].

Theorem 16 Suppose that a linear operator $A$ acts in the finite-dimensional space $\mathbb{R}^{N}$ and leaves a cone $K \subseteq \mathbb{R}^{N}$ invariant, i.e, $A K \subseteq K$. Then $\sigma(A)$ is an eigenvalue of $A$ and there is an eigenvector which corresponds to the eigenvalue $\sigma(A)$ and belongs to $K$.

The operator described in this last theorem is denoted by positive operator. For a given real number $a, \hat{K}(a)$ is a positive operator since for $X \in \mathbb{S}_{+}^{n}\left(\mathbb{R}, n_{q}\right)$, $\nu(Y)=\hat{K}(a) \nu(X), Y=\left(Y_{1}, \ldots, Y_{n_{q}}\right) \in \mathbb{S}_{+}^{n}\left(\mathbb{R}, n_{q}\right)$, where each $Y_{i}, i \in \mathcal{Q}$ is positive definite since it can be written as

$$
\begin{gathered}
\nu\left(Y_{i}\right)=\sum_{j=1}^{n_{q}} \hat{K}_{i, j}(a) \nu\left(X_{j}\right), \\
\hat{K}_{i, j}(a) \nu\left(X_{j}\right)=\nu\left(\sum_{l=1}^{n_{l}} \int_{0}^{T_{i, l}} E_{i, l}(s)^{\prime} X_{j} E_{i, l}(s) e^{-a s} \mathbf{1}_{\xi_{l}(i)=j} F_{i, l}(d s)\right)
\end{gathered}
$$

where we used (19) in obtaining (58). Note that $S_{+}^{n}\left(\mathbb{R}, n_{q}\right)$ can be embedded in $\mathbb{R}^{N}, N=n_{q} n^{2}$ and forms a cone in $\mathbb{R}^{N}$, in the sense that if $X, Y \in S_{+}^{n}\left(\mathbb{R}, n_{q}\right)$, then $\alpha_{1} X+\alpha_{2} Y \in S_{+}^{n}\left(\mathbb{R}, n_{q}\right), \forall_{\alpha_{1}, \alpha_{2} \geq 0}$.

Therefore, from Theorem 16 we conclude the following.

Corollary $\mathbf{1 7}$ For a given $a \in \mathbb{R}$ such that $\hat{K}(a)$ exists,

$$
\exists_{X \in \mathbb{S}_{+}^{n}\left(\mathbb{R}, n_{q}\right)}: \hat{K}(a) \nu(X)=\sigma(\hat{K}(a)) \nu(X) .
$$


The following result summarizes standard results of Laplace transforms of functions (cf. [13, Ch. 2,Th. 2.8]) and measures (cf. [13, Ch. 3,Th. 8.2])

Theorem 18 For a matrix-valued function $W(t)$ defined in $\mathbb{R}_{\geq 0}$ if $W(z):=$ $\int_{0}^{T} W(t) e^{-z t} F(d t)$ exists for a given real a, then it exists in $\Re[z] \geq a$ and it is continuous in this region and analytic in $\Re[z]>a$. The same is true if we replace $F(d t)$ by $d t$.

The following proposition establishes important properties of the function $\sigma(\hat{K}(y))$.

Proposition 19 Suppose that $a \in \mathbb{R}$ is such that $\hat{K}(a)$ exists. Then $\sigma(\hat{K}(y))$ is a continuous functions in $y \geq a$, and $\sigma(\hat{K}(y)) \rightarrow 0$ as $y \rightarrow+\infty$.

Proof. The fact that $\hat{K}(y)$ is a continuous function of $y \geq a$ follows from Theorem 18. The spectral radius of $\hat{K}(y)$ is continuous, since the function $\sigma(\hat{K}(y))=\max \left\{\left|\lambda_{i}(\hat{K}(y))\right|\right\}$ is a composite of continuous functions. In fact, the eigenvalues of a continuously parameter dependent matrix function are continuous functions of the parameter (cf. [11]). To prove the second part of the statement, notice that each block component of $\hat{K}(y)$ takes the form

$$
\begin{array}{r}
\hat{K}_{i, j}(y)=\sum_{l=1}^{n_{l}}\left[\int_{0}^{T_{i, l}}\left(E_{i, l}(s)^{\prime}\right)^{(2)} e^{-y s} \mathbf{1}_{\xi_{l}(i)=j} f_{i, l}(s) d s+\right. \\
\left.\sum_{i} w_{i}\left(E_{i, l}\left(b_{i}\right)^{\prime}\right)^{(2)} e^{-y b_{i}} \mathbf{1}_{\xi_{l}(i)=j}\right]
\end{array}
$$

Each second term inside the summation clearly tends to zero as $y \rightarrow+\infty$, and the fact that each first term tends to zero as $y \rightarrow+\infty$ is a general fact of Laplace transforms (cf., [13, Ch. 2, Th. 2.7]). Thus, $\sigma(\hat{K}(y))$ tends to zero as $y$ tends to infinite.

The following proposition can be derived from [17, Prop. 1].

Proposition 20 For $B \in \mathbb{C}^{n_{q} n^{2} \times n^{2}}$ the following are equivalent

(a) $\sigma(B)<1$,

(b) $\lim _{k \rightarrow+\infty} B^{k} \nu(X)=0$, for every $X \in S_{+}^{n}\left(\mathbb{C}, n_{q}\right)$.

We are ready to prove Theorems 13 and 14. For two elements of the space of $n_{q}$ complex matrices $X=\left(X_{1}, \ldots, X_{n_{q}}\right), X_{i} \in \mathbb{C}^{n \times n}, Y=\left(Y_{1}, \ldots, Y_{n_{q}}\right), Y_{i} \in$ $\mathbb{C}^{n \times n}, i \in \mathcal{Q}$, we consider the following inner product $\langle X, Y\rangle=\sum_{i=1}^{n_{q}} \operatorname{tr}\left(X_{i}^{\prime} Y_{i}\right)$. We

Proof. (of Theorem 13) From (57) we have that if $Y: \nu(Y)=\hat{K}(a) \nu(X)$ for $X \in S_{+}^{n}\left(\mathbb{C}, n_{q}\right)$ then $Y \in S_{+}^{n}\left(\mathbb{C}, n_{q}\right)$, and therefore by induction we conclude that if $Y: \nu(Y)=\hat{K}^{k}(a) \nu(X)$ for $X \in S_{+}^{n}(\mathbb{C})$ then $Y \in S_{+}^{n}\left(\mathbb{C}, n_{q}\right)$ and $k \in \mathbb{Z}_{>0}$. Note that such $Y=\left(Y_{1}, \ldots, Y_{n_{q}}\right): \nu(Y)=\hat{K}^{k}(a) \nu(X)$ can be written as a sum of terms taking the form 


$$
\begin{array}{r}
Y_{i}=\sum_{\substack{i_{1}, \ldots, i_{k}, l_{1}, \ldots, l_{k}, j \in \mathcal{Q}}} \int_{0}^{T_{i_{k} l_{k}}} \ldots \int_{0}^{T_{i_{1} l_{1}}} E_{i_{k}, l_{k}}\left(h_{k}\right)^{\prime} \ldots E_{i_{1}, l_{1}}\left(h_{1}\right)^{\prime} X_{j} E_{i_{1}, l_{1}}\left(k_{1}\right) \ldots \\
\ldots E_{i_{k}, l_{k}}\left(h_{k}\right) e^{-a h_{1}} \ldots e^{-a h_{k}} F_{i_{1} l_{1}}\left(d h_{1}\right) \ldots F_{i_{k} l_{k}}\left(d h_{k}\right),
\end{array}
$$

where $i \in \mathcal{Q}$ and the indexes $i_{1}, \ldots, i_{k}$ and $l_{1}, \ldots, l_{k}$, are determined by the maps $\xi_{l}$. Thus, for $W=\left(W_{1}, \ldots, W_{n_{q}}\right) \in S_{+}^{n}\left(\mathbb{C}, n_{q}\right),\langle W, Y\rangle$ can be written as a sum of terms taking the form

$$
\begin{gathered}
\langle W, Y\rangle=\sum_{\substack{i_{1}, \ldots i_{k}, r \in \mathcal{Q} \\
l_{1}, \ldots, l_{k}, j \in \mathcal{Q}}} \int_{0}^{T_{i_{k} l_{k}}} \ldots \int_{0}^{T_{i_{1} l_{1}}} g_{i_{1}, \ldots i_{k}, l_{1}, \ldots, l_{k}, r, j}\left(h_{1}, \ldots, h_{k}\right) \\
e^{-a h_{1}} \ldots e^{-a h_{k}} F_{i_{1} l_{1}}\left(d h_{1}\right) \ldots F_{i_{k} l_{k}}\left(d h_{k}\right),
\end{gathered}
$$

where

$$
\begin{aligned}
& g_{i_{1}, \ldots i_{k}, l_{1}, \ldots, l_{k}, r, j}\left(h_{1}, \ldots, h_{k}\right) \\
& \quad=\operatorname{tr}\left(W_{r}^{\prime} E_{i_{k}, l_{k}}\left(h_{k}\right)^{\prime} \ldots E_{i_{1}, l_{1}}\left(h_{1}\right)^{\prime} X_{j} E_{i_{1}, l_{1}}\left(h_{1}\right) \ldots E_{i_{k}, l_{k}}\left(h_{k}\right)\right),
\end{aligned}
$$

is a non-negative function since it can be written as the inner product of two (complex) positive semi-definite matrices.

Let $\nu^{-1}$ be such that $\nu^{-1}(\nu(Y))=Y$, for $Y=S_{+}^{n}\left(\mathbb{C}, n_{q}\right)$. For $z: \Re[z] \geq a$, and $W, X \in S_{+}^{n}\left(\mathbb{C}, n_{q}\right)$, we have

$$
\begin{aligned}
& \left|\left\langle W, \nu^{-1}\left(\hat{K}^{k}(z) \nu(X)\right)\right\rangle\right| \\
& =\mid \sum_{\substack{i_{1}, \ldots i_{k}, r \in \mathcal{Q} \\
l_{1}, \ldots, l_{k}, j \in \mathcal{Q}}} \int_{0}^{T_{i_{k} l_{k}}} \cdots \int_{0}^{T_{i_{1} l_{1}}} g_{i_{1}, \ldots i_{k}, l_{1}, \ldots, l_{k}, r, j}\left(h_{1}, \ldots, h_{k}\right) \\
& \quad e^{-z h_{1}} \ldots e^{-z h_{k}} F\left(d h_{1}\right) \ldots F\left(d h_{k}\right) \mid \\
& \leq \sum_{\substack{i_{1}, \ldots i_{k}, r \in \mathcal{Q} \\
l_{1}, \ldots, l_{k}, j \in \mathcal{Q}}} \int_{0}^{T_{i_{k} l_{k}}} \cdots \int_{0}^{T_{i_{1} l_{1}}}\left|g_{i_{1}, \ldots i_{k}, l_{1}, \ldots, l_{k}, r, j}\left(h_{1}, \ldots, h_{k}\right)\right| \\
& =\left\langle W, \nu^{-1}\left(\hat{K}^{k}(a) \nu(X)\right)\right\rangle
\end{aligned}
$$

where the integral interchanges from (61) to (62) and from (63) to (64) can be justified by the Fubini's Theorem and the equality between (63) and (64) is justified by the fact that we can remove the absolute value in (63) because $g_{i_{1}, \ldots i_{k}, l_{1}, \ldots, l_{k}, r, j}\left(h_{1}, \ldots, h_{k}\right)$ is a positive function.

If $\sigma(\hat{K}(a))<1$, by Proposition 20, $\hat{K}^{k}(a) \nu(X)$ tends to zero as $k$ tends to infinity and by the inequality (61)-(64) this implies that $\left|\left\langle W, \nu^{-1}\left(\hat{K}^{k}(z) \nu(X)\right)\right\rangle\right|$ tends to zero as $k$ tends to infinity, for any matrix $W \in S_{+}^{n}\left(\mathbb{C}, n_{q}\right)$. Given an arbitrary matrix $Z=\left(Z_{1}, \ldots, Z_{n_{q}}\right), Z_{1} \in \mathbb{C}^{n \times n n_{q}}$ we can write $Z=Z_{1}+Z_{2}+$ $i\left(Z_{3}+Z_{4}\right)$, where $Z_{i} \in S_{+}^{n}\left(\mathbb{C}, n_{q}\right)$ (cf. [17]), and therefore $\left|\left\langle Z, \nu^{-1}\left(\hat{K}^{k}(z) \nu(X)\right)\right\rangle\right|$ 
also tends to zero as $k$ tends to infinity, and this implies that $\hat{K}^{k}(z) \nu(X)$ tends to zero as $k$ tends to infinity. By Proposition 20, we conclude that $\sigma(\hat{K}(z))<1$.

To conclude the proof notice that for $\alpha>0$, if $\sigma(\hat{K}(a))<\alpha$ then $\sigma\left(\frac{1}{\alpha} \hat{K}(a)\right)<$ 1. Using the above arguments this implies that $\sigma\left(\frac{1}{\alpha} \hat{K}(z)\right)<1$, for $z: \Re[z] \geq a$, or equivalently that $\sigma(\hat{K}(z))<\alpha$. Since $\alpha$ is arbitrary, $\sigma(\hat{K}(z)) \leq \sigma(\hat{K}(a))$.

Proof. (of Theorem 14) We start by proving (i). If $\sigma(\hat{K}(a))=1$, from Corollary 17 , we have that 1 is an eigenvalue of $\hat{K}(a)$ and therefore $\operatorname{det}\left(I-\hat{K}\left(a_{1}\right)\right)=0$, for $a_{1}=a$. Suppose now that $\sigma(\hat{K}(a))>1$. By Proposition $19, \sigma(\hat{K}(y))$ is continuous along $y \geq a$ and tends to zero as $y \rightarrow+\infty$. Therefore, by the intermediate value theorem, $\exists_{a_{1}>0}: \sigma\left(\hat{K}\left(a_{1}\right)\right)=1$. Since, by Corollary $17, \sigma\left(\hat{K}\left(a_{1}\right)\right)=1$ is an eigenvalue, this implies that $\operatorname{det}\left(I-\hat{K}\left(a_{1}\right)\right)=0$. Moreover $a_{1}$ is the unique real number that verifies $\sigma\left(\hat{K}\left(a_{1}\right)\right)=1$. To see this, suppose that there exists another real number $a_{2}$ for which $\hat{K}\left(a_{2}\right)$ exists and $\sigma\left(\hat{K}\left(a_{2}\right)\right)=1$. In this case, due to the monotonicity of $\sigma(\hat{K}(y))$ for real $y$ established in Theorem 13, we must have $\sigma(\hat{K}(y))=1$ in $y \in\left[\min \left\{a_{1}, a_{2}\right\}, \max \left\{a_{1}, a_{2}\right\}\right]$. Since, by Corollary $17, \sigma\left(\hat{K}\left(a_{1}\right)\right)=1$ is an eigenvalue, this implies that $\operatorname{det}(I-\hat{K}(y))=0$, in $y \in\left[\min \left\{a_{1}, a_{2}\right\}, \max \left\{a_{1}, a_{2}\right\}\right]$. But $\operatorname{det}(I-\hat{K}(z))=0$ is analytic and the zeros of analytic zeros are isolated (cf. [18]), which leads to a contradiction.To prove (ii) note that, also by Corollary 17 , there exists $X \geq 0$ such that $\hat{K}\left(a_{1}\right) \nu(X)=\nu(X)$ which is (ii). Finally, we argue by contradiction to prove (iii). If there exists $\bar{z}$ verifying $\Re[\bar{z}]>a_{1}$ and $\operatorname{det}(I-\hat{K}(\bar{z}))=0$ then $\hat{K}(\bar{z})$ has an eigenvalue at 1 and therefore $\sigma(\hat{K}(\bar{z})) \geq 1$. From Theorem 13, $\sigma(\hat{K}(\bar{z})) \leq \sigma(\hat{K}(\Re[\bar{z}]))$ which implies that $\sigma(\hat{K}(\Re[\bar{z}])) \geq 1$. Now, $\sigma(\hat{K}(\Re[\bar{z}]))$ cannot be equal to one since $a_{1}$ is the unique real number which verifies this property and $\Re[\bar{z}]>a_{1}$. This leads to a contradiction since, again by Theorem 13, $\sigma(\hat{K}(\Re[\bar{z}])) \leq \sigma\left(\hat{K}\left(a_{1}\right)\right)=1$.

\section{A.3 Proofs of the main results}

Having proved Theorems 13, 14 and 15, we turn to the proof of Theorem 5. This requires the following theorem which can be concluded from $[13$, Thm. 4.9, Ch.4].

Theorem 21 Consider a Volterra equation taking the form (51) and suppose that $\hat{K}(0)$ exists. If

$$
\inf _{\Re[z] \geq 0}|\operatorname{det}(I-\hat{K}(z))|>0
$$

then:

(i) $\zeta(t)$ is bounded, continuous and tends to zero if $h(t)$ is bounded, continuous and tends to zero,

(ii) $\int_{0}^{\infty} \zeta(t) d t$ exists if $\int_{0}^{\infty} h(t) d t$ exists. 
Theorem 5 is established next assuming Theorems 8 and 9 .

Proof. (of Theorem 5)

Firstly, we prove that $\inf |\operatorname{det}(I-\hat{K}(z))|>0$ for $z$ in the closed complex right half plane is equivalent to $\operatorname{det}(I-K(z)) \neq 0$ in the same complex region. Necessity is obvious. To prove sufficiency we start by noticing that by Theorem $9, \operatorname{det}(I-\hat{K}(z)) \geq 0$ in the mentioned complex region is equivalent to $\sigma(M)<1$, where $M:=\hat{K}(0)$. If $\sigma(M)<1$, then by Theorem 13, $\sigma(\hat{K}(z))<1$ in the closed right half plane. This implies that $|\operatorname{det}(I-\hat{K}(z))|=$ $\Pi_{i=1}^{n^{2}}\left|1-\lambda_{i}(\hat{K}(z))\right| \geq(1-\sigma(M))^{n^{2}}$. Therefore $\inf (|\operatorname{det}(I-\hat{K}(z))|)>0$ holds in the closed right half plane.

Secondly, we prove that $(26)$ is equivalent to $h(t)$ tends to zero, $(27)$ is equivalent to $\int_{0}^{T} h(t) d t<\infty$ and (28) is equivalent to $h(t)$ converges to zero exponentially fast. To see this, for a given $i \in \mathcal{Q}$, consider the following Jordan normal form decomposition of $A_{i}=V \operatorname{diag}\left(\left[\begin{array}{ll}D_{\bar{\lambda}\left(A_{i}\right)} & D_{2}\end{array}\right]\right) W^{\prime}$, where $D_{\bar{\lambda}\left(A_{i}\right)}$ is any of the (possibly more than one) largest Jordan blocks associated with the eigenvalues with largest real part $\bar{\lambda}\left(A_{i}\right)$,

$$
V=\left[\begin{array}{lllll}
v_{1} & \ldots & v_{\bar{m}\left(A_{i}\right)} & v_{\bar{m}\left(A_{i}\right)+1} \ldots v_{n}
\end{array}\right]
$$

and

$$
W=\left(V^{-1}\right)^{\prime}=\left[\begin{array}{llll}
w_{1} & \ldots w_{\bar{m}\left(A_{i}\right)} & w_{\bar{m}\left(A_{i}\right)+1} \ldots w_{n}
\end{array}\right]
$$

Then $e^{A_{i} t}=v_{1} w_{\bar{m}\left(A_{i}\right)}^{\prime} e^{\bar{\lambda}\left(A_{i}\right) t} t^{\bar{m}\left(A_{i}\right)}+U_{i}(t)$ for a known function $U_{i}(t)$, and $h_{i}(t)=\nu\left(H_{i}(t), H_{i}(t)=\bar{H}_{i} e^{2 \bar{\lambda}\left(A_{i}\right) t} t^{2 \bar{m}\left(A_{i}\right)} S_{i}(t)+\tilde{H}_{i}(t)\right.$, where $\bar{H}_{i}=\left(v_{1}^{\prime} v_{1}\right) w_{\bar{m}\left(A_{i}\right)} w_{\bar{m}\left(A_{i}\right)}^{\prime}$ and the function $\tilde{H}_{i}(t)$ tends to zero, is integrable or tends to zero exponentially fast if the respective condition holds for $e^{2 \lambda\left(A_{i}\right) t} t^{2 \bar{m}\left(A_{i}\right)} S_{i}(t)$. Since $\bar{H}_{i} \neq 0$ for every $i \in \mathcal{Q}$, the desired conclusion follows.

Thirdly, note that since $\mathbb{E}\left[x(t)^{\prime} x(t)\right]=x_{0}^{\prime} Z(t) x_{0}$, MSS is equivalent to $Z(t) \rightarrow$ $0, \mathrm{SS}$ is equivalent to $\int_{0}^{\infty} Z(t) d t$ exists, and MES is equivalent to $Z(t)$ tends to zero exponentially fast. Sufficiency is obvious, and necessity follows from the fact that each $Z_{i}(t), i \in \mathcal{Q}$ is a positive semi-definite non-zero matrix since from (55) we conclude that it is the sum of positive semi-definite matrices, and one of these matrices $H(t)$ is non-zero.

Sufficiency of conditions (25) and (26) for MSS and of conditions (25) and (27) for SS follows then directly from Theorem 21. In fact, (25) is equivalent to $(65)$, and (i) since $H(t)$ is bounded and continuous and by (26) tends to zero, the same is true by Theorem 21 for $Z(t)$ (MSS), (ii) since $H(t)$ is integrable by $(27)$, the same is true by Theorem 21 for $Z(t)$ (SS).

To prove sufficiency of (25) and (28) for MES, we start by proving that (28) implies (T1). From the observation right before (29), it suffices to prove that (29) holds or equivalently that $\int_{0}^{T_{i, l}}\left(e^{2 \lambda s}-1\right) F_{i, l}(d s)<\infty$ for $\lambda>\bar{\lambda}_{i}(A), \forall_{i \in \mathcal{Q}, l \in \mathcal{L}}$. Integrating by parts, yields

$$
\int_{0}^{T_{i, l}}\left(e^{2 \lambda s}-1\right) F(d s)=\left.\left(e^{2 \lambda s}-1\right) S(t)\right|_{s=0} ^{s={ }^{T_{i}, l}}-\int_{0}^{T_{i, l}} 2 \lambda e^{2 \lambda s} S(t) d t .
$$

Choosing $\lambda=\max \left\{\bar{\lambda}\left(A_{i}\right)\right\}+\frac{\alpha_{1}}{4}$, from (28) we have that $e^{2 \lambda t} S(t) \leq c e^{-\frac{\alpha_{1}}{2} t}$ which implies that (66) is finite. Next, we consider a perturbation in the matrices $A_{i}, i \in \mathcal{Q}$ as in Theorem 15, i.e., $A_{\delta i}=A_{i}+\frac{\delta}{2} I$, and prove that for sufficiently small $\delta,(25)$ and $(28)$ still hold when we replace $\hat{K}(z)$ and $H(t)$ 
by $\hat{K}_{\delta}(z)$ and $H_{\delta}(t)$, respectively. From part (i) proved in the previous paragraph, this means that $Z_{\delta}(t) \rightarrow 0$, since (28) implies (26). Therefore, from Theorem 15, we conclude that $Z(t)=Z_{\delta}(t) e^{-\delta t}$, which means that $Z(t)$ tends to zero exponentially fast. To see that $\operatorname{det}\left(I-\hat{K}_{\delta}(z)\right) \neq 0$, in $\Re[z] \geq 0$, if $\operatorname{det}(I-\hat{K}(z)) \neq 0$, in $\Re[z] \geq 0$, notice that this latter condition is equivalent to $\sigma(\hat{K}(0))<1$ by Theorem 9. Using the continuity of $\sigma(\hat{K}(y))$ (Proposition 19) and using the fact that (T1) holds we conclude that for sufficiently small $\delta$, $\sigma(\hat{K}(-\delta))<1$. Using Theorem 15, we have $\sigma\left(\hat{K}_{\delta}(0)\right)=\sigma(\hat{K}(-\delta))<1$ which is equivalent to $\operatorname{det}\left(I-\hat{K}_{\delta}(z)\right) \neq 0$, in $\Re[z] \geq 0$. Finally, since (28) is equivalent to $H(t) \leq \bar{H} e^{-\alpha_{1} t}$ for some matrix $\bar{H}$, we have that $H_{\delta}(t)=H(t) e^{\delta t}$ tends to zero exponentially fast for $\delta<\alpha_{1}$.

Necessity of (26), (27), and (28), is proved by noticing that each $Z_{i}(t), i \in \mathcal{Q}$, described by (55), is a sum of positive semi-definite matrices, and therefore $Z(t) \geq H(t)$. Hence, $Z(t) \rightarrow 0$ (MSS), $Z(t)$ is integrable (SS), and $Z(t)$ tends to zero exponentially fast (MES), imply that the respectively condition must hold for $H(t)$ and therefore that (26), (27), and (28) must hold, respectively.

Necessity of (25) for MSS, SS and MES, assuming that (T1), (T2) and (28) hold, follows directly from Theorem 8 . In fact, if (25) does not hold, by Theorem $8, Z(t)$ tends to infinity exponentially fast, since $R_{1, j} \geq 0$ for some $j$.

If (28) does not hold, we can argue that $Z(t)$ does not tend to zero and is not integrable by considering a modified Volterra equation $Z_{1}(t)=K\left(Z_{1}(t)\right)+H_{1}(t)$, where $H_{1}(t):=H(t) e^{-c t}$ decreases exponentially fast for sufficiently large $c>0$. Therefore we can argue as in the last paragraph that $Z_{1}(t)$ does not verify (26), (27), or (28), and neither does $Z(t)$ since $Z(t) \geq Z_{1}(t)$. In fact, defining $O(t):=Z(t)-Z_{1}(t)$, we have that $O(t)$ verifies the Volterra equation $O(t)=$ $K(O(t))+H(t)-H_{1}(t)$, which has a positive semi-definite solution, similarly to $(55)$, since $H(t)-H_{1}(t) \geq 0$.

We prove next Theorem 7 .

Proof. (of Theorem 7)

We consider a perturbation in the matrices $A_{i}, i \in \mathcal{Q}$ as in Theorem 15, i.e., $A_{\delta i}=A_{i}+\frac{\delta}{2} I$, and prove that for $\delta<\alpha=\min \left\{\alpha_{1}, \alpha_{2}\right\},(25)$ and (28) still hold when we replace $\hat{K}(z)$ and $H(t)$ by $\hat{K}_{\delta}(z)$ and $H_{\delta}(t)$, respectively. From Theorem 5 this means that $Z_{\delta}(t)$ tends to zero exponentially fast, and from Theorem $15, Z(t)=Z_{\delta}(t) e^{-\delta t}$ tends to zero exponentially fast with the desired decay constant (and so does $\left.\mathbb{E}\left[x^{\prime}(t) x(t)\right]=x_{0}^{\prime} Z(t) x_{0}\right)$.

To see that (28) still holds for $H_{\delta}(t)$, notice that since (28) is equivalent to $H(t) \leq \bar{H} e^{\alpha_{1} t}$ for some matrix $\bar{H}$ (second paragraph of the proof of Theorem 5), we have that $H_{\delta}(t)=H(t) e^{\delta t}$ is exponentially bounded for $\delta<\alpha_{1}$.

To see that $(25)$ still holds for $\hat{K}_{\delta}(z)$, we distinguish two cases: the case where there exists $\bar{a}<0$ such that $\operatorname{det}(I-\hat{K}(\bar{a}))=0$ and when such $\bar{a}<0$ does not exist. In the first case, $\hat{K}(\bar{a})$ has an eigenvalue equal to 1 and therefore $\sigma(\hat{K}(\bar{a})) \geq 1$. From Theorem 14, there exists $a \geq \bar{a}$ such that $\operatorname{det}(I-\hat{K}(a))=0$, and if $z$ is such that $\operatorname{det}(I-\hat{K}(z))=0$ then $\Re[z] \leq a$. Thus, $\operatorname{det}\left(I-\hat{K}_{\delta}(z)\right)=$ $\operatorname{det}(I-\hat{K}(z-\delta)) \neq 0$ in $\Re[z] \geq 0$ for $\delta<\bar{a}=\alpha_{2}$. In the second case, we prove that $\operatorname{det}(I-\hat{K}(z)) \neq 0$ in $\Re[z]<0$, and therefore this condition is met in the whole complex plane since the SHS is MES, which means by Theorem 5 that (25) must hold. Arguing by contradiction, if there is a $\bar{z}<0$ such that $\operatorname{det}(I-\hat{K}(\bar{z}))=0, \hat{K}(\bar{z})$ has an eigenvalue equal to 1 and therefore $\sigma(\hat{K}(\bar{z})) \geq 1$. By Theorem 13, $\sigma(\hat{K}(\Re[z])) \geq \sigma(\hat{K}(z)) \geq 1$ and by Theorem 14 there exists 
$a>\Re[z]$ such that $\operatorname{det}(I-\hat{K}(a))=0$ which leads to a contradiction since $a$ cannot be strictly negative by hypothesis and cannot be positive because the SHS is MES. In this case, we can choose $\delta$ arbitrarily large, since for any $\delta$, $\operatorname{det}\left(I-\hat{K}_{\delta}(z)\right)=\operatorname{det}(I-\hat{K}(z-\delta)) \neq 0$ in $\Re[z] \geq 0$.

To prove Theorem 8 we need the following Theorem which can be derived from [13, Th. 2.4 and 2.5 , Ch. 7]. We say that a matrix-valued measure $\rho(d t)$ is finite (in $\mathbb{R}_{\geq 0}$ ) if $\int_{0}^{\infty} \rho(d t)<\infty$, and denote its Laplace transform by $\hat{\rho}(z):=\int_{0}^{\infty} e^{-z t} \rho(d t)$.

Theorem 22 Consider the Volterra equation (51) and suppose that $\hat{K}(0)$ exists, and

$$
\begin{gathered}
\inf _{z: \Re[z]=0}|\operatorname{det}(I-\hat{K}(z))|>0, \\
\lim _{|z| \rightarrow \infty, \Re[z] \geq 0} \inf |\operatorname{det}(I-\hat{K}(z))|>0 .
\end{gathered}
$$

Then there exists a finite number of $z_{i}$ such that $\operatorname{det}\left(I-\hat{K}\left(z_{i}\right)\right)=0$ and the solution to (51) takes the form

$$
\zeta(t)=h(t)+\int_{0}^{t} \rho(d s) h(t-s)
$$

where

$$
\rho(d s)=\sum_{i=1}^{n_{z}} \sum_{j=0}^{m_{i}-1} S_{i, j} s^{j} e^{z_{i} s} d s+\xi(d s),
$$

and $\xi(d s)$ is a finite measure. Moreover, $\hat{\rho}(z)=\hat{K}(z)[I-\hat{K}(z)]^{-1}$ and the matrices $S_{i, j}$ are such that in a neighborhood of $z_{i}$ we have

$$
\hat{\rho}(z)=\sum_{j=0}^{m_{i}-1} S_{i, j} \frac{j !}{\left(z-z_{i}\right)^{j+1}}+\zeta_{i}(z),
$$

where $\zeta_{i}(z)$ are analytic matrix-valued functions.

Proof. (of Theorem 8)

Due to (T1) and Theorem $18, \hat{K}(z)$ is analytic in $\Re[z]>-\epsilon$ for some $\epsilon>0$. To prove (i) we argue (as in [13, pp.192]) that the existence of an infinite number of zeros of the analytic function $\operatorname{det}(I-\hat{K}(z))$ in $\Re[z]>-\epsilon$ would imply that there exists a sequence of zeros of $\operatorname{det}(I-\hat{K}(z))$ which either converges to a finite zero $z_{0}$ of $\operatorname{det}(I-\hat{K}(z))$, or tends to infinity. The first cannot happen for $z_{0}$ in the interior of $\Re[z]>-\epsilon$ because $\operatorname{det}(I-\hat{K}(z))$ is analytic in this region and the zeros of analytic functions are isolated (cf. [18]). If $z_{0}$ is in the boundary, i.e., $\Re\left[z_{0}\right]=-\epsilon$ we can reduce $\epsilon$, i.e., consider $\bar{\epsilon}<\epsilon$ for which there is a finite number of zeros in $\Re[z]>-\bar{\epsilon}$. The second cannot happen due to (T2)(cf. [13, pp.195]).

To prove (ii) notice that from the equivalence between (A) and (B) of Theorem 9 we must have $\sigma(\hat{K}(0)) \geq 1$. Thus, from Theorem 14 there exists $a_{1}=z_{1}>0$ with the desired properties.

The first part of the statement (iii) follows from Theorem 22 provided that we can bypass conditions (68) and (67). Notice that (T2) implies (68). Since 
we assume (T2), the condition (67) can be simply stated as the characteristic equation $\operatorname{det}(I-\hat{K}(z))=0$ has no zeros on the imaginary axis (non-critical case). In this non-critical case, from (69) and (70) we can conclude that

$$
\zeta(t)=\sum_{i=1}^{n_{z}} \sum_{j=0}^{m_{i}-1} r_{i, j} t^{j} e^{z_{i} t} d t+y(t)
$$

(cf. $[13$, Th. 2.5, Ch.7]) where

$$
r_{i, j}=\sum_{k=0}^{m_{i}-j-1}\left(\begin{array}{c}
j+k \\
j
\end{array}\right) S_{i, j+k} \hat{H}^{(k)}\left(z_{l}\right),
$$

$\hat{H}^{(k)}\left(z_{l}\right)$ denotes the $k$ th derivative, and $y(t):=\int_{0}^{t} \xi(d s) h(t-s)+h(t)$ tends to zero as $t \rightarrow \infty$ if $H^{\nu}(t)$ tends to zero as $t \rightarrow \infty$. This is the case since we assume (28). We will prove shortly that $y(t)$ tends to zero exponentially fast. The matrices $r_{i, j}$ are also determined by (30) since applying Laplace transforms on both sides of (69), we obtain

$$
\hat{Z}(z)=\hat{H}(z)+\hat{\rho}(z) \hat{H}(z)=[I-\hat{K}(z)]^{-1} \hat{H}(z)
$$

where $\hat{Z}(z):=\int_{0}^{\infty} \zeta(t) e^{-z t} d t$ and $\hat{H}(z):=\int_{0}^{\infty} h(t) e^{-z t} d t$, and applying Laplace transforms on both sides of (72) we obtain

$$
\hat{Z}(z)=\sum_{i=1}^{n_{z}} \sum_{j=0}^{m_{i}-1} r_{i, j} \frac{j !}{\left(z-z_{i}\right)^{j+1}}+\hat{Y}(z),
$$

where $\hat{Y}(z):=\int_{0}^{\infty} y(t) e^{-z t} d t$. By uniqueness of the Laplace transform (74) and (75) must be equal. Notice that $\hat{y}(z)$ is analytic because $y(t)$ is integrable (since it tends to zero exponentially fast) and the Laplace transform of an integrable function is analytic in its region of convergence.

If $\operatorname{det}(I-\hat{K}(z))$ has zeros on the imaginary axis (critical case), i.e., (67) does not hold, we consider a perturbation on the matrices $A_{i}, i \in \mathcal{Q}$ as in Theorem 15, taking the form $A_{\delta i}=A_{i}+\frac{\delta}{2} I$. The argument is that there exists $\delta<\epsilon$, where $\epsilon$ is the constant in (T1), such that the characteristic equation of the perturbed Volterra equation does not have zeros on the imaginary axis. In fact, let $\left\{\tilde{z}_{i}, i \in\left\{1, \ldots, \tilde{n}_{z}\right\}\right\}$ be the possible zeros of $\operatorname{det}(I-\hat{K}(z))$ in $-\epsilon<\Re[z]<0$, which exist in a finite number due to part (i) of the present Theorem. Since by Theorem $15, \hat{K}_{\delta}(z)=\hat{K}(z-\delta)$, choosing $\delta<\epsilon$ such that $\delta \neq \Re\left[\tilde{z}_{i}\right]$ for $1 \leq i \leq \tilde{n}_{z}$, we have $\operatorname{det}\left(I-\hat{K}_{\delta}(z)\right)=\operatorname{det}(I-\hat{K}(z-\delta)) \neq 0$ in $\Re[z]=0$. Thus, the $\delta$-perturbed Volterra equation satisfies the conditions of Theorem 22. We can then apply Theorem 22 and obtain that

$$
z_{\delta}(t)=\sum_{i=1}^{n_{z}} \sum_{j=0}^{m_{i}-1} r_{\delta i, j} t^{j} e^{\left(z_{i}+\delta\right) t}+\sum_{i=1}^{\bar{n}_{z}} \sum_{j=1}^{\bar{m}_{i}} \tilde{r}_{\delta i j} t^{j} e^{\bar{z}_{i} t}+y_{\delta}(t)
$$

where $\bar{z}_{i}$ are the (possible) zeros of $\operatorname{det}\left(I-\hat{K}_{\delta}(z)\right)$ in $0<\Re\left[\bar{z}_{i}\right]<\delta$, and $r_{\delta i, j}$ are determined by the residues of $\left[I-\hat{K}_{\delta}(z)\right]^{-1} \hat{H}_{\delta}(z)$ at $z_{i}+\delta$. Since $\hat{H}_{\delta}(z)=$ $\int_{0}^{\infty} H^{\nu}(t) e^{\delta t} e^{-z t} d t=\hat{H}(z-\delta)$ and, from Theorem $15, \hat{K}_{\delta}(z)=\hat{K}(z-\delta)$, we conclude that the residues of $\left[I-\hat{K}_{\delta}(z)\right]^{-1} \hat{H}_{\delta}(z)=[I-\hat{K}(z-\delta)]^{-1} \hat{H}(z-\delta)$ at 
$z_{i}+\delta$ equal the residues of $[I-\hat{K}(z)]^{-1} \hat{H}(z)$ at $z_{i}$. Thus, $r_{\delta i, j}=r_{i, j}$. Since the solution to the original Volterra equation is $\zeta(t)=\zeta_{\delta}(t) e^{-\delta t}$, it takes the form

$$
\zeta(t)=\sum_{i=1}^{n_{z}} \sum_{j=0}^{m_{i}-1} r_{i, j} t^{j} e^{z_{i} t}+\tilde{\zeta}(t),
$$

where $\tilde{\zeta}(t)$ tends to zero exponentially fast.

Notice that also in the non-critical case we can choose $\delta<\epsilon$, where $\epsilon$ is the constant in (T1), and applying the same argument as in the critical case show that $y(t)$ tends to zero exponentially fast.

Finally, we prove that $r_{1, j}$ cannot be zero for all $j$. If this was the case, $z_{1}$ would be a removable singularity of the function $\hat{R}(z) \hat{H}(z)$ where $\hat{R}(z):=$ $(I-\hat{K}(z))^{-1}$ and therefore $\lim _{z \rightarrow z_{1}} \hat{R}(z) \hat{H}(z)$ would be finite (cf. [18]). We prove that this leads to a contradiction. We establish first that $\sigma(\hat{K}(a))<\sigma\left(\hat{K}\left(z_{1}\right)\right)=$ 1 , for real $a>z_{1}$. To see this, notice that by Theorem 13, $\sigma(\hat{K}(a)) \leq \sigma\left(\hat{K}\left(z_{1}\right)\right)$. If equality would hold, i.e., $\sigma(\hat{K}(a))=1$, due to the monotonicity of $\sigma(\hat{K}(y))$ for real $y$, we would have $\sigma(\hat{K}(y))=1$ in $y \in\left[z_{1}, a\right]$. Since, by Corollary 17, $\sigma(\hat{K}(y))=1$ is an eigenvalue, this would imply that $\operatorname{det}(I-\hat{K}(y))=0$, in $y \in\left[\min \left\{a_{1}, a_{2}\right\}, \max \left\{a_{1}, a_{2}\right\}\right]$. But $\operatorname{det}(I-\hat{K}(z))=0$ is analytic and the zeros of analytic zeros are isolated (cf. [18]), which leads to a contradiction. Since $\sigma(\hat{K}(a))<\sigma\left(\hat{K}\left(z_{1}\right)\right)=1$, we can expand $\hat{R}(a)$ as a von Neumann series and obtain $\hat{R}(a) \hat{H}(a)=\sum_{k=0}^{\infty} \hat{K}^{k}(a) \hat{H}(a)$. From the characterization of $z_{1}$ in Theorem 14 we have that there exists $X \in S_{+}^{n}\left(\mathbb{C}, n_{q}\right)$ such that $\hat{K}\left(z_{1}\right)(\nu(X))=$ $\nu(X)$. Since

$$
\begin{aligned}
& \nu^{-1}(\hat{H}(y))= \\
& \left(\int_{0}^{\infty} e^{A_{1}^{\prime} s} e^{A_{1} s} S_{1}(t) e^{-y s} d s, \ldots, \int_{0}^{\infty} e^{A_{n_{q}}^{\prime} s} e^{A_{n_{q}} s} S_{n_{q}}(t) e^{-y s} d s\right) \in S_{+}^{n}\left(\mathbb{C}, n_{q}\right),
\end{aligned}
$$

there exists $\alpha$ such that $\nu^{-1}(\hat{H}(y)) \geq \alpha X$. Notice that since $\hat{K}(a)$ is a positive operator, in the sense explicit in (57), by induction $\hat{K}^{k}(a)$ is also a positive operator. Thus, $\nu^{-1}\left(\hat{K}^{k}(a)(\hat{H}(a)-\alpha \nu(X))\right) \geq 0$ or equivalently $\nu^{-1}\left(\hat{K}^{k}(a) \hat{H}(a)\right) \geq$ $\alpha \nu^{-1}\left(\tilde{K}^{k}(a) \nu(X)\right)$ which implies that $\nu^{-1}(\hat{R}(a) \hat{H}(a)) \geq \alpha \nu^{-1}(\hat{R}(a)(\nu(X)))$. But $\hat{R}(a)(\nu(X))$ tends to infinite as $x$ tends to $z_{1}$ for values $x>z_{1}$ since $\lim _{x \rightarrow z_{1}, x>z_{1}} \hat{R}(a) \nu(X)=\lim _{a \rightarrow z_{1}, a>z_{1}} \sum_{k=0}^{\infty} \hat{K}(a) \nu(X)=\sum_{k=1}^{\infty} \hat{K}\left(z_{1}\right) \nu(X)=$ $\sum_{k=1}^{\infty} \nu(X)$. Thus, $\hat{R}(a) \hat{H}(a) \rightarrow \infty$ as $a \rightarrow z_{1}, a>z_{1}$. This means that $z_{1}$ is not a removable singularity, because if this was the case this limit would have to be finite.

Proof. (of Theorem 9)

(B) $\Rightarrow(\mathrm{A})$ From Theorem 13, $\sigma(\hat{K}(z)) \leq \sigma(\hat{K}(0))$ for any $z: \Re[z] \geq 0$. This implies that if (B) holds, all the eigenvalues of $K(z)$ have a modulus strictly less than one, and therefore $\operatorname{det}(I-\hat{K}(z))$ cannot be zero in $\Re[z] \geq 0$, which is (A). $(\mathrm{A}) \Rightarrow(\mathrm{B})$ If $\sigma(\hat{K}(0)) \geq 1$, from Theorem 14 we have that there exists $a_{1} \geq 0$ such that $\operatorname{det}\left(I-\hat{K}\left(a_{1}\right)\right)=0$, and therefore condition (A) does not hold at $z=a_{1}$.

$(B) \Rightarrow(D)$ The linear system of equations (32) can be written in the vector form 


$$
\hat{K}(0)\left[\begin{array}{c}
\nu\left(P_{1}\right) \\
\vdots \\
\nu\left(P_{n_{q}}\right)
\end{array}\right]=\left[\begin{array}{c}
\nu\left(Q_{1}\right) \\
\vdots \\
\nu\left(Q_{n_{q}}\right)
\end{array}\right]
$$

If $\sigma(\hat{K}(0))<1$ this system of equations has a unique solution given by $P=$ $\sum_{k=0}^{+\infty} L^{k}(Q)$, where $P=\left(P_{1}, \ldots, P_{n_{q}}\right), Q=\left(Q_{1}, \ldots, Q_{n_{q}}\right)$, and $L(Q):=$ $\left(L_{1}(Q), \ldots, L_{n_{q}}(Q)\right)$. The solution of this system of equation verifies $P \geq 0$ if $Q \geq 0$ due to the fact that $L$ is a positive operator (i.e., $Q \geq 0$ implies $L(Q) \geq 0)$. If $Q>0, P=Q+\sum_{k=1}^{+\infty} L^{k}(Q) \geq Q>0$.

$(\mathrm{D}) \Rightarrow(\mathrm{C})$ For any $Q:=\left(Q_{1}, \ldots, Q_{n_{q}}\right)>0$, the matrix $P:=\left(P_{1}, \ldots, P_{n_{q}}\right)>0$ which verifies $(\mathrm{D})$ also verifies $(\mathrm{C})$.

$(\mathrm{C}) \Rightarrow(\mathrm{B})$ If $(\mathrm{C})$ holds, then $L(P)-P=-S<0$ for some $S=\left(S_{1}, \ldots, S_{n_{q}}\right)>0$. Rewriting this expression as $P=S+L(P)$ we conclude that for any positive $k$ we have $P=L^{k}(P)+\sum_{j=0}^{k-1} L^{j}(S)$. Letting $k$ tend to infinity we conclude that $\sum_{j=0}^{\infty} L^{j}(S)$ must be bounded, which implies that $L^{k}(S) \rightarrow 0$ as $k \rightarrow \infty$. Given any $X \in S_{+}^{n}\left(\mathbb{C}, n_{q}\right)$ there exists $\alpha>0$ such that $S-\alpha X \in S_{+}^{n}\left(\mathbb{C}, n_{q}\right)$. Using the fact that $L^{k}(S)-\alpha L^{k}(X)=L^{k}(S-\alpha X) \in S_{+}^{n}\left(\mathbb{C}, n_{q}\right)$ (because $L$ is a positive operator), we conclude that $L^{k}(X)=\nu^{-1}\left(\hat{K}(0)^{k} \nu(X)\right) \rightarrow 0$ as $k \rightarrow \infty$ since $L^{k}(S) \rightarrow 0$ as $k \rightarrow \infty$. Thus, $\sigma(\hat{K}(0))<1$ by Theorem 16 .

Finally, we prove the Proposition 6.

Proof. (of Proposition 6) The condition (T1) assures that $\hat{K}_{d}(y)$ exists for $y \geq-\epsilon$. In this region, $\sigma\left(\hat{K}_{d}(y)\right)$ is continuous as established in Proposition 19. Therefore, $\sigma\left(\hat{K}_{d}(0)\right)<1$ implies that $\sigma\left(\hat{K}_{d}\left(-\epsilon_{1}\right)\right)<1$ for some $\epsilon_{1} \in(0, \epsilon)$. Applying Theorem 13 in the case where the distribution $F=F_{d}$ is purely discrete, we conclude that $\sigma\left(\hat{K}_{d}\left(-\epsilon_{1}\right)\right)<1$ implies that $\sigma\left(\hat{K}_{d}(z)\right)<1$ in $\Re[z] \geq$ $-\epsilon_{1}$, which encompasses $\mathcal{C}\left(\epsilon_{1}, R\right)$ for any $R>0$. Therefore, the modulus of the eigenvalues of $\hat{K}_{d}(z)$ in $\mathcal{C}\left(\epsilon_{1}, R\right)$ is strictly less than one in $\Re[z] \geq-\epsilon_{1}$, which implies that $\inf \left|\operatorname{det}\left(I-\hat{K}_{d}(z)\right)\right|>0$ in $\mathcal{C}\left(\epsilon_{1}, R\right)$.

\section{References}

[1] G. Pola, M. Bujorianu, J. Lygeros, and M. D. Benedetto, "Stochastic hybrid models: An overview," in IFAC Conference on Analysis and Design of Hybrid Systems. St. Malo: Springer, 2003, pp. 16-18.

[2] J. Hu, J. Lygeros, and S. Sastry, "Towards a theory of stochastic hybrid systems," in In Third International Workshop on Hybrid Systems: Computation and Control. Springer, 2000, pp. 160-173.

[3] M. K. Ghosh, A. Arapostathis, and S. I. Marcus, "Ergodic control of switching diffusions," SIAM Journal of Control and Optimization, vol. 35, pp. 1952-1988, Nov. 1997.

[4] M. H. A. Davis, Markov Models and Optimization. London, UK: Chapman \& Hall, 1993.

[5] J. P. Hespanha, "A model for stochastic hybrid systems with application to communication networks," Nonlinear Analysis, Special Issue on Hybrid Systems, vol. 62, no. 8, pp. 1353-1383, Sep. 2005. 
[6] H. Blom and J. Lygeros, Stochastic Hybrid Systems: Theory and Safety Critical Applications. Lecture Notes in Control and Information Sciences, Springer-Verlag, 2006.

[7] J. Lygeros, "Stochastic hybrid systems: Theory and applications," in Control and Decision Conference, 2008. CCDC 2008. Chinese, July 2008, pp. $40-42$.

[8] M. Mariton, Jump Linear Systems in Automatic Control. New York, NY, USA: Marcel Dekker Inc, 1990.

[9] Janssen, Jacques, Manca, and Raimondo, Applied Semi-Markov Processes. Springer-Verlag, 2006.

[10] D. Antunes, J. P. Hespanha, and C. Silvestre, "Analysis of impulsive renewal systems: Application to emulation in networked control," Sep. 2009, submitted.

[11] R. A. Horn and C. R. Johnson, Topics in matrix analysis. New York, NY, USA: Cambridge University Press, 1994.

[12] P. Linz, Analytical and Numerical Methods for Volterra Equations. SIAM Studies in Applied Mathematics 7, 1985.

[13] G. Gripenberg, S. O. Londen, and O. Staffans, Volterra Integral and Functional Equations. Cambridge University Press, 1990.

[14] D. Antunes, J. P. Hespanha, and C. Silvestre, "Stochastic hybrid systems with renewal transitions," Sep. 2009, internal report.

[15] G. F. Franklin, D. J. Powell, and A. Emami-Naeini, Feedback Control of Dynamic Systems. Upper Saddle River, NJ, USA: Prentice Hall PTR, 2001.

[16] M. A. Krasnoselskii, J. A. Lifshits, and A. V. Sobolev, Positive linear systems : the method of positive operators. Berlin: Heldermann, Verlag, 1989.

[17] C. Kubrusly and O. Costa, "Mean square stability conditions for discrete stochastic bilinear systems," Automatic Control, IEEE Transactions on, vol. 30, no. 11, pp. 1082-1087, Nov 1985.

[18] W. Rudin, Real and complex analysis, 3rd ed. New York, NY, USA: McGraw-Hill, Inc., 1987. 